\title{
Systematic classification of two-loop realizations of the Weinberg operator
}

\author{
D. Aristizabal Sierra, ${ }^{a}$ A. Degee, ${ }^{a}$ L. Dorame ${ }^{b}$ and M. Hirsch ${ }^{b}$ \\ ${ }^{a}$ IFPA, Dep. AGO, Universite de Liege, \\ Bat B5, Sart Tilman B-4000 Liege 1, Belgium \\ ${ }^{b}$ AHEP Group, Instituto de Fisica Corpuscular-C.S.I.C./Universitat de Valencia, \\ Edificio Institutos de Paterna, Apt 22085, E-46071 Valencia, Spain \\ E-mail: daristizabal@ulg.ac.be, audrey.degee@ulg.ac.be, \\ dorame@ific.uv.es, mahirsch@ific.uv.es
}

ABSTRACT: We systematically analyze the $d=5$ Weinberg operator at 2-loop order. Using a diagrammatic approach, we identify two different interesting categories of neutrino mass models: (i) Genuine 2-loop models for which both, tree-level and 1-loop contributions, are guaranteed to be absent. And (ii) finite 2-loop diagrams, which correspond to the 1-loop generation of some particular vertex appearing in a given 1-loop neutrino mass model, thus being effectively 2-loop. From the large list of all possible 2-loop diagrams, the vast majority are infinite corrections to lower order neutrino mass models and only a moderately small number of diagrams fall into these two interesting classes. Moreover, all diagrams in class (i) are just variations of three basic diagrams, with examples discussed in the literature before. Similarly, we also show that class (ii) diagrams consists of only variations of these three plus two more basic diagrams. Finally, we show how our results can be consistently and readily used in order to construct two-loop neutrino mass models.

Keywords: Beyond Standard Model, Neutrino Physics

ARXIV EPRINT: 1411.7038 


\section{Contents}

1 Introduction 1

2 Two-loop 1PI topologies, diagrams, genuine models and quantum numbers

2.1 Generalities, strategy and notation $\quad 6$

$\begin{array}{lll}2.2 & \text { Two-loop 1PI topologies } & 7\end{array}$

2.3 Constructing diagrams 8

$\begin{array}{ll}2.4 \text { Genuine diagrams } & 10\end{array}$

$\begin{array}{ll}2.5 & \text { SM gauge quantum numbers } \\ & 14\end{array}$

$\begin{array}{ll}\text { 2.5.1 Assigning quantum numbers: some examples } & 17\end{array}$

3 Constructing two-loop models $\quad 18$

$\begin{array}{lll}3.1 & \text { Genuine 2-loop models } & 18\end{array}$

3.2 Non-genuine but finite 2-loop models 22

4 Conclusions $\quad 25$

A Non-renormalizable topologies and finite non-genuine diagrams $\quad 26$

$\begin{array}{ll}\text { B Quantum numbers } & 27\end{array}$

C Useful formulas for 2-loop calculations $\quad 28$

\section{Introduction}

Neutrino masses observed in oscillation experiments [1-3] are so far the only signal for physics beyond the standard model (SM) measured in laboratories. However, while we do know now mass squared differences and mixing angles to a very high precision [4-6], there is no experimental data on whether neutrinos are Dirac or Majorana particles.

From the low energy point of view Majorana neutrino masses are described by a leptonnumber-breaking dimension five effective operator, known as the Weinberg operator [7]:

$$
\mathcal{O}_{5}=\frac{c_{\alpha \beta}}{\Lambda}\left(\overline{L_{\alpha}^{c}} i \tau_{2} H\right)\left(H^{T} i \tau_{2} L_{\beta}\right)
$$

The smallness of the observed light neutrino masses can then be explained from eq. (1.1) as being due to either a large scale $\Lambda$ or a small coffecient $c_{\alpha \beta}$ (or both). However, disentangling these possibilities requires going beyond this effective operator picture.

It is well-known that at tree-level only three UV completions for the Weinberg operator exist [8]: these are usually called type-I [9-12], type-II [13-16] and type-III [17] seesaw. All 
of them have in common that for $c_{\alpha \beta} \simeq \mathcal{O}\left(10^{-2}-1\right), \Lambda \simeq 10^{13-15} \mathrm{GeV}$ is needed to produce sub-eV neutrino masses. Thus, while being an attractive possibility from the theoretical point of view, experimentally the classical seesaws do not offer any possible tests - apart from neutrino masses themselves and the fact that neutrinos are predicted to be Majorana particles, thus a finite rate for $0 \nu \beta \beta$ decay should exist.

However, $c_{\alpha \beta}$ could easily be smaller than $\mathcal{O}(1)$. Essentially there are three possibilities to arrange this:

1. The neutrino mass is generated at tree level, but an additional suppression enters through a small lepton-number-violating (LNV) coupling. The so-called "inverse" seesaw [18] or "linear" seesaw [19, 20] are examples for this approach.

2. The neutrino mass is generated radiatively. The additional suppression is guaranteed by a combination of loop integrals and sub-EW scale masses (for example SM charged lepton masses) entering the diagrams. At the one- and two-loop level, the Zee $[21]^{1}$ and the Cheng-Li-Babu-Zee models stand as benchmark references [16, 26, 27] and probably due to this reason they have been the subject of intensive phenomenological studies [25, 28-34].

3. The neutrino mass is forbidden at $d=5$, but appears from effective operators of higher dimension $[35,36]$. Such an approach is not feasible in models with only the SM Higgs doublet, since $\left(H^{\dagger} H\right)$ is a complete singlet and can not carry any charges. ${ }^{2}$ However, in a two-Higgs doublet world (or more complicated setups) forbidding the $d=5$ while allowing $d=7$ could be realized with, for example, the help of some flavor symmetry that prevents the direct Yukawa coupling of the SM Higgs doublet to the light fermions.

In this paper we will focus on the second possibility: loop neutrino masses. In [38] the Weinberg operator was studied systematically at the one-loop level. Two topologies (for a total of four diagrams) were identified to give neutrino masses at the 1-loop level genuinely (i.e. without producing neutrino masses at tree-level), see figure 1. Three more diagrams were found, that can be understood as 1-loop realizations of one of the known tree-level seesaws and the relation between tree- and 1-loop diagrams were discussed. In our current work, we extend this analysis [38] to the 2-loop level, following the same diagrammaticbased approach.

The systematic decomposition used in [38] allows one to identify all possible realizations of $\mathcal{O}_{5}$ at a given loop level, in principle. However, while there are only 12 diagrams (out of which only seven turn out to be of any interest) at the 1-loop level, at the 2-loop level one can naively expect to find order $\mathcal{O}(100)$ diagrams, which need to be studied. We have followed therefore a sort of "algorithm" for $\mathcal{O}_{5}$ at the two-loop order: $(i)$ derive all possible

\footnotetext{
${ }^{1}$ The minimal Zee model [22] is ruled out since it predicts maximal mixing in the atmospheric as well as in the solar sector. However, its non-minimal version is fully consistent with neutrino data [23-25].

${ }^{2}$ Note that an exception to this statement does exist. If the UV completion involves higher $\mathrm{SU}(2)$ representations (quadruplet scalar and triplet fermion), then a model generating at the effective level the $d=7$ effective operator $L H L H H^{\dagger} H$ can be written [37].
} 

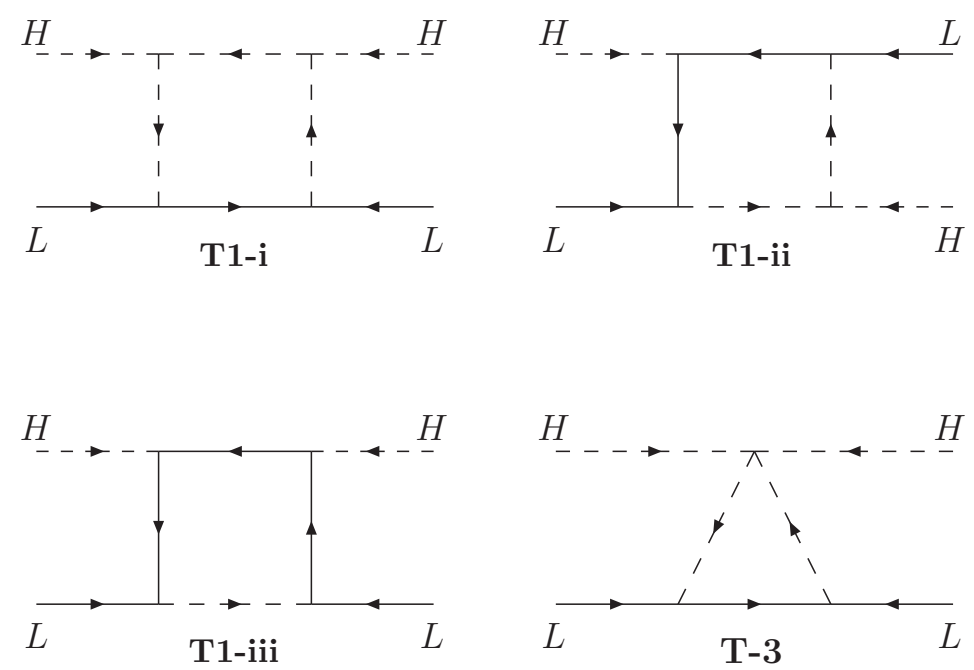

Figure 1. The four diagrams leading to genuine 1-loop neutrino mass models. The notation of [38] is used to classify these diagrams. Just to mention two examples: diagram T1-ii corresponds to the the classical Zee model [21], while an example for T-3 is the "scotogenic" model of [39].

two-loop topologies; exclude all 1-loop reducible topologies from this list. (ii) into the remaining topologies insert fermions and scalars, to create all possible diagram variations; exclude from further analysis all those diagrams which need non-renormalizable vertices. For these first two steps we have extensively used FeynArts [40], in order to ensure that no topology is missed. (iii) Identify in this list of diagrams (with renormalizable vertices only) all those, for which no 1-loop diagram (nor a tree-level neutrino mass) exist. We call these diagrams "genuine 2-loop diagrams" and classify them as class-I diagrams (or models). (iv) For all remaining diagrams one can then distinguish diagrams which lead to finite loop integrals from those with inifinite integrals. The former cases, which are our "class-II" diagrams, can present interesting models of neutrino mass, even though they are not genuinely 2-loop. The characterization of class-II diagrams (and their corresponding models) is similar to the discussion given in [38] for the 1-loop order: class-II diagrams can give a theoretical motivation for the smallness of a particular vertex, generated at 1-loop order. This particular vertex then appears in one of the four genuine 1-loop neutrino mass diagrams (see figure 1), making the whole construction effectively 2-loop. Diagrams with infinite loop integrals, on the other hand, can never lead to interesting models and can therefore be discarded.

Surprisingly, the result of the above exercise allows one to show that in the moderate number of diagrams of class-I all cases are variations of only three basic diagrams, two of which have been known in the literature for a long time: the Cheng-Li-Babu-Zee [16, 26, 27] diagram (CLBZ in the following) and another similar diagram first considered in two independent papers by Petcov and Toshev [41] and by Babu and Ma [42] (PTBM in the following). The third basic diagram we call the "rainbow" diagram (RB in the following). Similarly, it can be shown that all diagrams in class-II can be described by variations of just five basic types of diagrams: we call them the non-genuine CLBZ and PTBM and RB diagrams plus two internal scalar correction diagrams (two categories, called ISC-i and ISC-ii). 
Before entering into the details, let us mention that our study considers only scalar bosons, while, for example, the original papers on the PTBM diagram [41, 42] use the SM $W$-boson. We decided to concentrate on scalars for essentially two reasons: (a) From a topological point of view, diagrams with scalar or vector bosons are equivalent. Thus, from our list of diagrams for scalars the corresponding diagrams for vectors can be easily derived. ${ }^{3}$ And (b) apart from the few cases with SM $W$-bosons, new vector-mediated cases require that the vector should be a gauge boson under a new symmetry, and the mass should be given by the spontaneous breaking of that symmetry. This means that the scalar sector of the model needs to be discussed as well; see [43] for a recent example.

Our list of diagrams allows us to recover 2-loop models discussed previously in the literature. Apart from the standard diagrams CLBZ [16, 26, 27] and PTBM [41, 42] in their original incarnations (enumerated as CLBZ-1 and PTBM-1 in the following), ${ }^{4}$ we have found a number of variations of these genuine diagrams and also several realizations of our class-II diagrams have been discussed in the literature. For example, a variant of CLBZ-1 with an additional neutral scalar vev to generate the lepton number violating triple scalar vertex $h^{-} h^{-} k^{++}$has been discussed in [44, 45]. A supersymmetric extension of CLBZ has been discussed in [46]. A new model with a scalar diquark and a scalar leptoquark has been discussed in [47], 2-loop neutrino masses are generated by the CLBZ-1 diagram. Ref. [48] considers a model with neutrino masses due to CLBZ-1 and a $\mathbb{Z}_{3}$ symmetry to eliminate tree-level seesaw and also explain dark matter. There are also models in the literature based on other variants of CLBZ. CLBZ-3 appears in [49], CLBZ-9 in [50, 51] and CLBZ-8 and CLBZ-10 appear within the 331-model of [52]. A possible connection between two-loop neutrino masses and dark matter has been explored in $[53,54]$ in two models giving each a CLBZ-3 type diagram. Then there are also models, based on CLBZ, using vectors instead of scalars [55-57]. All these models are realizations of a 2-loop gauge-mediated diagram involving an internal effective coupling (see e.g. [58]). In refs. $[55,56]$ this effective coupling is generated at the tree level via the mixing of an $\mathrm{SU}(2)$ scalar triplet with a doubly charged singlet, thus resulting in a 2-loop model (effectively CLBZ-9). Note that this construction requires that the tree-level coupling between the triplet and the leptons is absent. Ref. [59], instead, discusses the case where the effective coupling is induced at the 1-loop order, thus leading to a 3-loop gauge-mediated neutrino mass model. Finally, ref. [57] considers a partial UV completion involving an effective lepton number-violating vertex for $W$ bosons with a doubly charged singlet. Then there are models based on variants of PTBM such as [60], which uses leptoquarks and a colour octet fermion. Also in R-parity violation PTBM diagrams appear [61] and can be used to constrain the R-parity violating soft SUSY breaking parameters. Such R-parity violating SUSY models have not only PTBM diagrams, but also RB type 2-loop diagrams [62]. Then there are leptoquark models [63] and extensions of the SM with vector-like quarks [64], with scalar and SM $W$ boson diagrams. In this case, both CLBZ and PTBM in various variants contribute to the neutrino mass. RB diagrams

\footnotetext{
${ }^{3}$ Of course, the propagator of a massive vector boson is different from that of a scalar. Thus, the expressions for the 2-loop integrals need to be modified accordingly.

${ }^{4}$ The numbers of the variants quoted correspond to those given in figures 4,5 and figures $16,17,18$ and 19 in appendix A.
} 
where considered, for example, in $[65,66]$. However, those models contain RB-diagrams of higher order, $\left[(L H)^{2} S^{2}\right] / \Lambda^{3}$, and thus do not fall into our classification scheme. The 1-loop diagram T-3, see figure 1, contains a quartic scalar vertex, usually its parameter is called $\lambda_{5}$. The radiative generation of $\lambda_{5}$ for this diagram, via diagrams of class ISC-i has been considered in [67]. Similarly ISC-i variant-5 was discussed in [68].

On top of these "pure" 2-loop models, also mixed situations, where one (or more) neutrinos have tree-level masses, while one neutrino mass is generated at 2-loop level have been considered. Ref. [69] considers such a situation, with some neutrinos getting a mass through CLBZ-1. Similarly, [70] assumes two neutrino masses to be tree-level and calculates the minimal mass for the remaining neutrino, generated through diagrams with Higgses of the form PTBM-1 in both SM and MSSM. Ref. [71] considers a variant with some neutrinos receiving 1-loop neutrino masses and others are 2-loop. Also, [72] consider models where neutrino mass appear at 1-loop level and also at 2-loop level with CLBZ-1, PTBM-4 and two variants of the RB diagram. ${ }^{5}$

We mention that there are also papers on two-loop models in the literature, which are not covered by our classification, because they are of higher dimension than the Weinberg operator. To quote two more examples, the papers [73-76] have several variants of CLBZ at $d=7$, while [77] has a variant of the RB at $d=7$. Finally there is the paper [78], that discusses Dirac neutrino masses at 2-loop.

Our work is, of course, not the first attempt to organize neutrino mass models systematically. Apart from the above-mentioned paper [38], which treats the 1-loop case, a set of "rules and recipes" for neutrino masses at 1-loop, 2-loop and higher orders has been discussed in [79] and our approach has some overlap with this paper, too. Then, there is the interesting work of [80], which writes down all lepton number violating operators from $d=5$ to $d=11$. Decomposing these operators, one can find a list of tree-level, 1-loop, 2-loop etc. diagrams, which allow to specify neutrino mass models [80-84]. Our study is complementary to the analysis done in these papers in that it provides further insight for the specific two-loop case, exhaustively listing all possible diagrams. However, different from [80-84], we put quite some emphasis on our classification schemes, which allow us to distinguish "genuine" models, i.e. those for which the absence of 1-loop masses is guaranteed from the "non-genuine" (or class-II) models.

The rest of this paper is organized as follows. In section 2.1 we discuss the "strategy" followed in this paper and introduce our notation. Section 2.2 is devoted to the classification of relevant topologies. "Genuine" and "non-genuine" diagrams are discussed next and SM electroweak-sector quantum number assignments are given. In section 3 we exemplify the use of our results by constructing two specific examples of UV completions. In section 4 we summarize and present our conclusions. The bulk of the technical details of our calculation is collected in appendices A, B and C, where we list renormalizable topologies leading to non-genuine finite and infinite diagrams, non-renormalizable topologies, as well as nongenuine diagrams, tables with the different quantum number assignments and relevant formulas for the evaluation of two-loop integrals.

\footnotetext{
${ }^{5}$ The diagrams shown for the RB are NG-RB-2 and one diagram with an infinite integral. The latter is, of course, not an interesting 2-loop model, but a correction to the 1-loop diagram.
} 


\section{Two-loop 1PI topologies, diagrams, genuine models and quantum num- bers}

\subsection{Generalities, strategy and notation}

A systematic classification of the two-loop order realizations of $\mathcal{O}_{5}$ using the diagrammatic method does so far not exist. The underlying reason is probably related with the fact that tackling the problem via the diagram-based method turns out to be challenging, due to the large number of two-loop diagrams, and unless precise guidelines are followed such study is not possible. Thus, in this section we discuss some generic guidelines that will allow us to deal with the 2-loop classification of $\mathcal{O}_{5}$.

At the 2-loop order, the dimension five effective operator consist of a set of topologies: $\mathcal{O}_{5}^{2-\text { loop }}=\left\{T 2_{1}, T 2_{2}, \ldots, T 2_{r}\right\}$. We have identified 29 distinct topologies (see below) out of which only a subset turns out to be relevant. Once all topologies have been identified, the next step is then that of specifying the fermion and scalar internal lines $(F$ and $S$ ) as well as the external lines ( $L$ and $H$ ) of each topology, i.e. "promoting" topologies to diagrams. Here, renormalizability fixes possible vertices to only dimension-four three and four point vertices (3-PVs and 4-PVs). Due to the freedom when placing the two external $L$ and $H$ lines, however, in general, a given two-loop order topology $T 2_{i}$ can involve quite a few number of Feynman diagrams. At this point it is possible to discard 11 topologies (see figure 15), since these will always lead to non-renormalizable diagrams, see appendix A.

From the remaining 18 topologies (see figures 2 and 14) only a subset leads to genuine two-loop diagrams. In order to identify non-genuine diagrams, one can assign arbitrary quantum numbers $q_{i}\left(q_{i}\right.$, related with a new symmetry or in some cases with the gauge symmetry itself, e.g. hypercharge) to the external and internal fields, and then enforce conservation of these charges vertex by vertex. These conservation rules define a set of conditions (denoted by $C^{2_{i}}$ ) that whenever satisfied guarantee the presence of the corresponding diagram. These conditions should be confronted with those arising from the 1-loop diagrams shown in figure 1 (denoted by $C^{1_{i}}$ ). Thus, if $C^{2_{i}} \subset C^{1_{i}}$, the corresponding 2-loop diagram will be necessarily accompanied by a 1-loop diagram, hence being nongenuine. Diagrams for which $C^{2_{i}} \subset C^{1_{i}}$ is not satisfied are potentially genuine, but their particle content must satisfy further constraints which guarantee the absence of both tree and 1-loop level diagrams (see section 2.4 for more details). Once these constraints are assured, the full list of truly genuine diagrams is fixed

Genuine diagrams define a set of renormalizable vertices, which will lead to a 2-loop UV completion (Lagrangian) once the gauge quantum numbers of the beyond SM fermion and scalar fields are specified. For that purpose the lepton and Higgs gauge quantum numbers can be used to constrain the possible quantum numbers of the internal fermion and scalar fields. This procedure, however, provides an unambiguous determination only in the case of trilinear couplings involving two SM fields. Let us discuss this in more detail. Yukawa (or pure scalar trilinear) couplings can involve two, one or none SM fields, schematically: $\bar{F} L H ; \bar{F} L S$ (which reduces to $\overline{L^{c}} L S$ whenever $F=L^{c}$ ), $\bar{F}_{1} F_{2} H ; \bar{F}_{1} F_{2} S$. In the first case, clearly $F$ has to be - unambiguously - a SU(2) singlet or triplet (type-I 
or type-III seesaws) while in the other cases $\mathrm{SU}(2) \times \mathrm{U}(1)_{Y}$ invariance requires:

$$
\begin{aligned}
n_{\bar{F}} \otimes n_{s} & \supset 2, & Y_{\bar{F}}+Y_{S}+Y_{L} & =0, \\
n_{F_{1}} \otimes n_{F_{2}} & \supset 2, & Y_{\bar{F}_{1}}+Y_{F_{2}}+Y_{H} & =0, \\
n_{F_{1}} \otimes n_{F_{2}} & \supset n_{\bar{S}}, & Y_{\bar{F}_{1}}+Y_{F_{2}}+Y_{S} & =0,
\end{aligned}
$$

where $n_{X}$ corresponds to the $\mathrm{SU}(2)$ representation under which the $X$ field transforms. From (2.1), it can be seen that - in principle - an infinite number of SU(2) representations as well as hypercharge assignments are consistent with the constraints implied by the lepton and Higgs quantum numbers. 4-PVs allow even for more freedom. These vertices can involve three, two, one or none SM fields (Higgses), schematically: $H H H S_{1}, H H S_{1} S_{2}$, $H S_{1} S_{2} S_{3}, S_{1} S_{2} S_{3} S_{4}$. So, in this case gauge invariance implies:

$$
\begin{aligned}
& n_{S_{1}}=\overline{4}, \\
& 3 Y_{H}+Y_{S_{1}}=0, \\
& n_{S_{1}} \otimes n_{S_{2}} \supset \overline{3}, \\
& 2 Y_{H}+Y_{S_{1}}+Y_{S_{2}}=0 \text {, } \\
& n_{S_{1}} \otimes n_{S_{2}} \otimes n_{S_{3}} \supset 2, \\
& Y_{H}+\sum_{i} Y_{S_{i}}=0 \text {, } \\
& n_{S_{1}} \otimes n_{S_{2}} \otimes n_{S_{3}} \otimes n_{S_{4}} \supset 1, \\
& \sum_{i} Y_{S_{i}}=0,
\end{aligned}
$$

which shows that apart from $H H H S_{1}$, there is no coupling which allows for an unambiguous determination of the SM gauge quantum numbers of the new fields.

Since trilinear couplings involving two SM fields are only possible in tree level realizations, the discussion above implies that once going beyond the tree level, a given genuine diagram leads to an infinite number of UV completions. From the practical point of view nevertheless one can impose an upper limit on the dimensions of the representations used. In our tables we list all combinations with singlets, doublets and triplets of $\mathrm{SU}(2)_{L}$. Tables for larger representations can be easily derived. We also mention that we do not give explicitly color quantum numbers in our tables since, as pointed out in [38], the inclusion of color is straightforward, see also the discussion in section 2.5.

Finally, once the UV completions are identified the only step which remains to be done is the determination of the light neutrino mass matrix, which requires calculating 2-loop integrals. Although the list of genuine diagrams is large this does not means that the number of 2-loop integrals to be evaluated is large. Different diagrams, not necessarily arising from the same topology, can involve the same 2-loop integral, essentially because after electroweak symmetry breaking the couplings to Higgs legs are just couplings to a background field: if coupled to fermions (scalars) they imply chirality flips (scalar mixing). This observation allows to reduce the number of integrals to be evaluated to just combinations of a few basic integrals, which we list in appendix C.

\subsection{Two-loop 1PI topologies}

Following the strategy described in section 2.1, our starting point consist in determining the complete set of two-loop one-particle irreducible (1PI) inequivalent topologies. At the two-loop order this set is expected to be large, so in order to generate an exhaustive list 


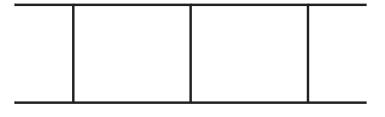

$T 2_{1}^{\mathrm{B}}$

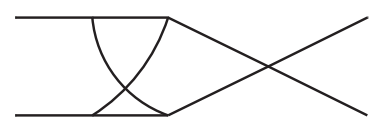

$T 2_{1}^{\mathrm{T}}$

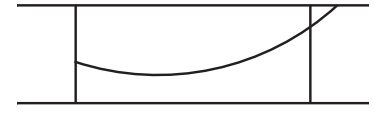

$T 2_{2}^{\mathrm{B}}$

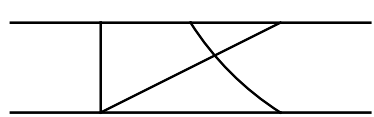

$T 2_{2}^{\mathrm{T}}$

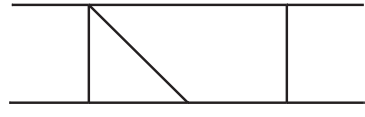

$T 2_{3}^{\mathrm{B}}$

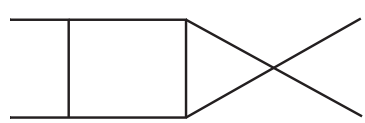

$T 2_{3}^{\mathrm{T}}$

Figure 2. 1PI box-based (upper row) and triangle-based (lower row) two-loop topologies, which can lead to genuine 2-loop models. All other topologies can lead only to class-II models, infinite renormlizations of 1-loop diagrams or contain non-renormalizable vertices. Those topologies are given in the appendix A.
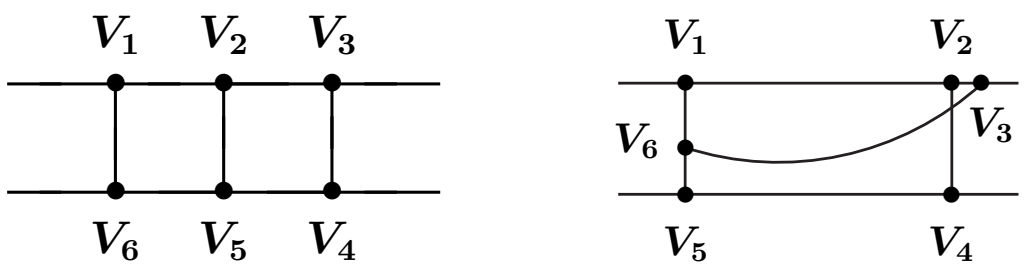

Figure 3. Vertex assignments for the two-loop $(6,0)$ topologies $T 2_{1}^{\mathrm{B}}$ and $T 2_{2}^{\mathrm{B}}$.

we proceed with FeynArts [40]. To simplify the output we suppress from the calculation topologies involving external legs self-energies, tadpoles and one-particle-reducible 2-loop topologies. The complete set of topologies is displayed in figure 2 and figures 14 and 15 in the appendix, respectively. In total we have identified 29 topologies, but only topologies listed in figure 2 lead to genuine diagrams. Topologies shown in figure 14 will lead to class-II models, while figure 15 shows for completeness the non-renormalizable topologies.

Denoting by (\#3-PVs, \#4-PVs) the number of 3-PVs and 4-PVs entering in each diagram, all topologies can be placed in four non-overlapping sets: $(2,2),(4,1),(6,0)$ and $(0,3)$. Since $4 \mathrm{PVs}$ are only possible for scalars, topologies not satisfying the renormalizability criterion should arise from the sets $(2,2)$ and $(0,3)$ (those involving the largest number of $4 \mathrm{PVs})$. Indeed, the set of topologies not satisfying this criterion consist of the full $(0,3)$ subset and eight $(2,2)$ topologies (see figure 15 ).

\subsection{Constructing diagrams}

No matter the topology, any diagram can be constructed from the following schematic Lagrangian:

$$
\begin{aligned}
\mathcal{L}= & Y_{L} \bar{L} F_{i} S_{k}+Y_{H} \bar{F}_{i} F_{j} H+\mu_{H} S_{i} S_{j} H+\lambda_{H} S_{i} S_{j} S_{k} H \\
& +\lambda_{H H} S_{i} S_{j} H H+Y \bar{F}_{i} F_{j} S_{k}+\mu_{s} S_{i} S_{j} S_{k}+\text { H.c. }
\end{aligned}
$$

In order to illustrate the method we have used for constructing diagrams we focus on the first two box-based topologies in figure $2\left(T 2_{1}^{B}\right.$ and $\left.T 2_{2}^{B}\right)$, and base our discussion on the diagrams sketched in figure 3. 


\begin{tabular}{|c|c|c|c|c|c|c|}
\hline$V_{1}$ & $V_{2}$ & $V_{3}$ & $V_{4}$ & $V_{5}$ & $V_{6}$ & Diagram \\
\hline \multirow{7}{*}{$L S F$} & \multirow{3}{*}{$F S F$} & $F S L$ & $H S S$ & $S S S$ & $S S H$ & CLBZ-3 \\
\hline & & \multirow{2}{*}{$F F H$} & $L F S$ & $S S S$ & $S S H$ & CLBZ-2 \\
\hline & & & $H F F$ & $F S F$ & $F S L$ & RB-1 \\
\hline & \multirow{4}{*}{$F F S$} & \multirow{2}{*}{$S F L$} & $H F F$ & $F F S$ & $S S H$ & PTBM-2 \\
\hline & & & $L F S$ & $S F F$ & $F S L$ & $x$ \\
\hline & & \multirow{2}{*}{$S S H$} & $H S S$ & $S F F$ & $F S L$ & RB-2 \\
\hline & & & $L S F$ & $F F S$ & $S S H$ & РTBM-3 \\
\hline \multirow{8}{*}{$L F S$} & \multirow{4}{*}{$S F F$} & \multirow{2}{*}{$F F H$} & $L F S$ & $S F F$ & $F F H$ & PTBM-1 \\
\hline & & & $H F F$ & $F F S$ & $S F L$ & DIV \\
\hline & & \multirow{2}{*}{$F S L$} & $L S F$ & $F F S$ & $S F L$ & $x$ \\
\hline & & & $H S S$ & $S F F$ & $F F H$ & РTBM-2 \\
\hline & \multirow{4}{*}{$S S S$} & \multirow{2}{*}{$S F L$} & $L F S$ & $S S S$ & $S F L$ & $x$ \\
\hline & & & $H F F$ & $F S F$ & $F F H$ & CLBZ-1 \\
\hline & & \multirow{2}{*}{$S S H$} & $L S F$ & $F S F$ & $F F H$ & CLBZ-2 \\
\hline & & & $H S S$ & $S S S$ & $S F L$ & ISC-i-3 \\
\hline
\end{tabular}

Table 1. Sequential vertex insertions leading to the full set of diagrams for topology $T 2_{1}^{\mathrm{B}}$. For $\boldsymbol{V}_{\mathbf{1}}, \boldsymbol{V}_{\mathbf{2}}, \boldsymbol{V}_{\mathbf{3}}$ the field sequence goes from left to right while for $\boldsymbol{V}_{\mathbf{4}}, \boldsymbol{V}_{\mathbf{5}}, \boldsymbol{V}_{\mathbf{6}}$ from right to left. Crosses indicate diagrams that do not correspond to $\mathcal{O}_{5}^{2-\text { loop }}$, while DIV a diagram involving a 2-loop divergent integral, hence of no interest.

In the $(6,0)$ case, external vertices always involve $Y_{L}, Y_{H}$ or $\mu_{H}$ couplings. So, in order to find an exhaustive list of possible Feynman diagrams one can start by fixing any of these couplings at $\boldsymbol{V}_{\mathbf{1}}$ (see figure 3 ) and then inserting sequentially in clockwise direction all possible vertices combinations. Table 1 illustrates the procedure for topology $T 2_{1}^{\mathrm{B}}$, where we have fixed at $\boldsymbol{V}_{\mathbf{1}}$ the $Y_{L}$ coupling. It can be seen that out of the 15 diagrams, 3 are possible only for four fermion external legs and so have nothing to do with $\mathcal{O}_{5}^{2-\text { loop }}$. In addition two diagrams appear twice (CLBZ-2 and PTBM-2), so at the end the 2-loop boxbased topology $T 2_{1}^{\mathrm{B}}$ involves 10 diagrams. For topology $T 2_{2}^{\mathrm{B}}$, determining the complete list requires considering at $\boldsymbol{V}_{\mathbf{1}}$ not only $Y_{L}$ but also $Y_{H}$ and $\mu_{H}$, due to its non-symmetric character. By doing so, the resulting list involves repeated diagrams (redundant diagrams) whose identification turns out to be complex. For that aim it is therefore useful to introduce the following sextuplet

$$
\left(n_{L}, n_{3}, n_{H}, n_{Y}, n_{S}, n_{4}\right)
$$


where the different entries label the number of $Y_{L}, \mu_{H}, Y_{H}, Y, \mu_{s}$ and $\lambda_{H}$ vertices defining a given diagram, and are such that depending on the topology obey certain constraints. For $(6,0)$-based diagrams these constraints read:

$$
\begin{aligned}
& n_{L}+n_{3}+n_{H}+n_{Y}+n_{S}+n_{4}=6, \\
& n_{L}=2, \quad n_{3}+n_{H}=2, \quad n_{4}=0, \quad n_{Y}+n_{S}=2,
\end{aligned}
$$

thus implying that the sextuplet structure of any diagram arising from $(6,0)$ topologies will necessarily belong to one of the following nine sextuplets:

$$
\begin{array}{lll}
(2,2,0,2,0,0), & (2,2,0,0,2,0), & (2,2,0,1,1,0), \\
(2,0,2,2,0,0), & (2,0,2,0,2,0), & (2,0,2,1,1,0), \\
(2,1,1,2,0,0), & (2,1,1,0,2,0), & (2,1,1,1,1,0) .
\end{array}
$$

For $T 2 \frac{\mathrm{B}}{2}$, the procedure outlined above yields 22 diagrams which can be grouped in five sets: one $(2,2,0,0,2,0)$, six $(2,2,0,1,1,0)$, five $(2,0,2,2,0,0)$, six $(2,1,1,2,0,0)$ and four $(2,1,1,1,1,0)$. Possible redundant diagrams should belong to a specific set, however since not all diagrams belonging to a given set are redundant, the identification of superfluous diagrams requires labelling the fermion and scalar lines of all diagrams within each set and comparing the different couplings. If the couplings of a couple of diagrams match, those diagrams count as one. So, proceeding in that way we have found that the number of diagrams arising from the $T 2_{2}^{\mathrm{B}}$ is ten.

Following this procedure for the remaining 16 topologies we have found the full set of diagrams for 1PI 2-loop topologies.

\subsection{Genuine diagrams}

Having identified all possible diagrams, we are now in a position to build the full list of genuine diagrams. The procedure to be followed involves two steps. First, we assume that the lepton and Higgs $\mathrm{SU}(2)$ doublets as well as the heavy fields flowing in the loops carry arbitrary charges $q_{i}$, and impose $q_{i}$ charge conservation vertex by vertex, as outlined in section 2.1 (and exemplified below). By doing so, we identify the non-genuine diagrams in our list. We are then left with diagrams which potentially lead to genuine models, see figure 4 and 5 . Their genuineness can then be guaranteed provided their particle content obeys the additional constraints discussed near the end of this section.

Let us first illustrate the $q_{i}$ charge procedure we have employed to identify non-genuine diagrams. The example we discuss is based on the 1- and 2-loop diagrams displayed in figure 6.

For diagram T1-i, the equations for $q_{i}$ conservation can be written as

$$
\begin{aligned}
q_{L}+q_{3} & =q_{4}, q_{L}+q_{4}=q_{1}, \\
q_{H}+q_{2} & =q_{1}, q_{H}+q_{3}=q_{2} .
\end{aligned}
$$

For diagram T-3 one has:

$$
q_{L}+q_{3}=q_{1}, \quad 2 q_{H}+q_{2}=q_{1}, \quad q_{L}+q_{2}=q_{3} .
$$




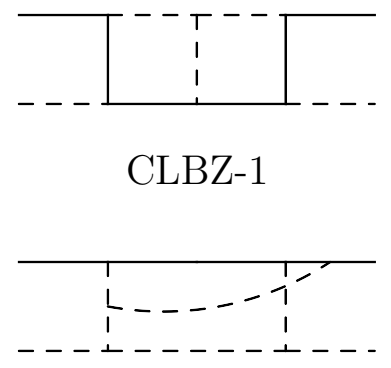

CLBZ-4

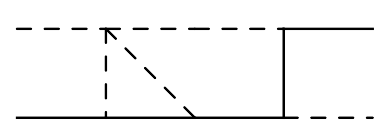

CLBZ-7

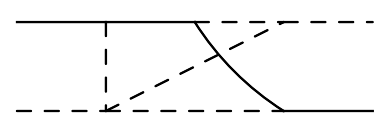

CLBZ-10

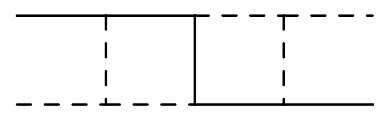

PTBM-3

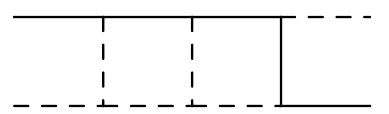

CLBZ-2

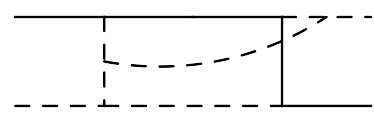

CLBZ-5

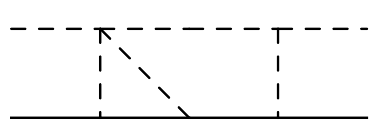

CLBZ-8

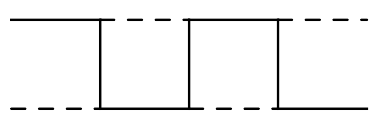

PTBM-1

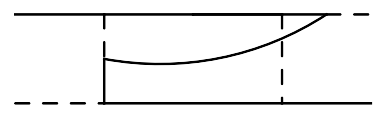

PTBM-4

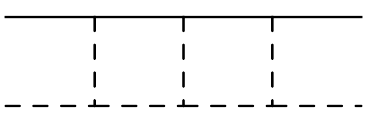

CLBZ-3

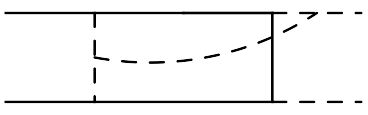

CLBZ-6

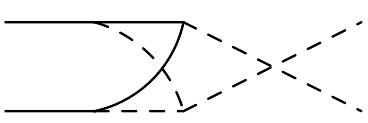

CLBZ-9

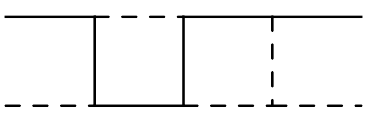

PTBM-2

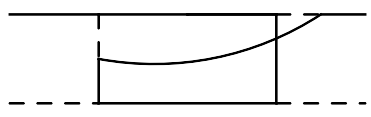

PTBM-5

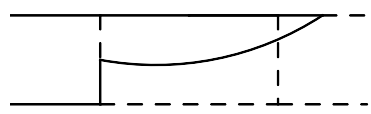

PTBM-6

Figure 4. Genuine two-loop CLBZ (Cheng-Li-Babu-Zee) and PTBM (Petcov-Toshev-Babu-Ma) diagrams arising from the topologies in figure 2. See the text for further details.

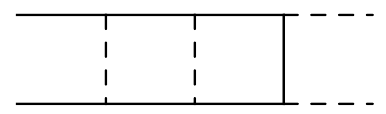

RB-1

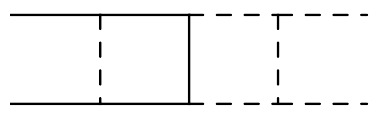

RB-2

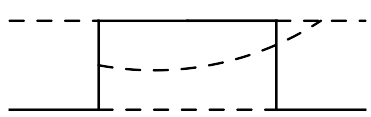

RB-3

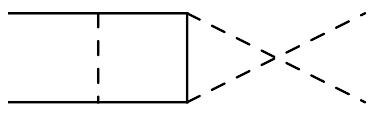

RB-4

Figure 5. Genuine two-loop RB diagrams. See the text for further details. 


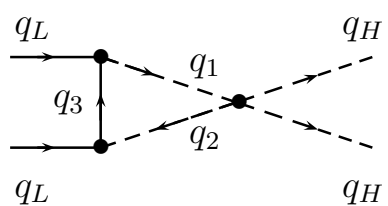

T-3

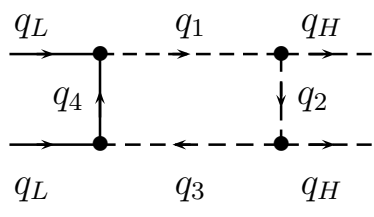

T1-i

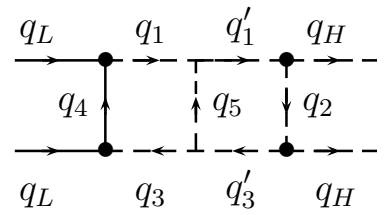

ISC-i-3

Figure 6. Charge $q_{i}$ flow for 1PI one-loop box and triangular diagrams (T1-i and T-3) as well as for the two-loop box-based diagram ICS-i-3.

The solution of these system of equations then leads to the following charge constraints:

$$
\begin{array}{lll}
C^{\mathrm{T} 1-\mathrm{i}}: & q_{4}=q_{3}+q_{L}, & q_{1}=q_{3}+2 q_{L}, \\
C^{\mathrm{T}-3}: & q_{1}=q_{2}+2 q_{L}, & q_{3}=q_{2}+q_{L},
\end{array}
$$

where $C^{\text {T1-i }}$ is the solution for the diagram T1-i in figure 6 , whereas $C^{\mathrm{T}-3}$ the solution for the one in eq. (2.9).

The constraints in (2.10) and (2.11) are to be used to know whether the 2-loop boxbased diagram ISC-i-3 in figure 6 is non-genuine or not. For that aim the $q_{i}$ charge conservation equations for this diagram has to be written. Conservation of charges implies:

$$
\begin{aligned}
q_{L}+q_{4} & =q_{1}, & q_{1}+q_{5} & =q_{1}^{\prime}, \\
q_{H}+q_{2} & =q_{1}^{\prime}, & q_{H}+q_{3}^{\prime} & =q_{2}, \\
q_{5}+q_{3} & =q_{3}^{\prime}, & q_{L}+q_{3} & =q_{4},
\end{aligned}
$$

and their solution is given by

$$
C^{\mathrm{ISC}-\mathrm{i}-3}: \quad q_{1}=q_{3}+2 q_{L}, \quad q_{4}=q_{3}+q_{L}, \quad q_{2}=q_{4}+q_{5}
$$

Comparing this solution with $C_{R}^{\text {T1-i }}$ in $(2.10)$, one can see that $q_{5} \neq 0$ forbids the 1-loop box-based diagram T1-i (right-hand side in figure 6). However, when comparing with $C^{\text {T-3 }}$ (trading $q_{2} \rightarrow q_{3}$ and $\left.q_{3} \rightarrow q_{4}\right)$ in (2.11) it is clear that constraints $C^{\text {ISC-i-3 }}$ allow the 1-loop triangle-based diagram T-3, independent of the choice of charges. One can then conclude that ISC-i-3 is not a genuine diagram.

Following this procedure, we have identified all non-genuine diagrams. These emerge from the topologies in figure 14 in appendix A. Moreover, we have found that the nongenuine but finite diagrams all belong to one of the following five different types, namely: (a) non-genuine CLBZ (NG-CLBZ), (b) non-genuine PTBM (NG-PTBM), (c) non-genuine 
RB (NG-RB), (d) ISC-i, (e) ISC-ii. Figures 16-19 in appendix A show this complete list of non-genuine but finite diagrams.

Let us now turn to the remaining (potentially) genuine diagrams that can not be eliminated after this procedure has been applied to the full list of diagrams. These are given in figure 4 and figure 5. All of these fall, as already stressed above, into the three classes: CLBZ, PTBM and RB. There is one subtlety involved in these RB diagrams, which we want to discuss briefly: in all non-genuine diagrams, see appendix A, it is possible to make a cut in the diagram, such that the remaining sub-diagram is equivalent to a vertex correction. Looking superficially to the RB diagrams in figure 5, it seems that such a cut is possible too, with the remaining sub-diagram being a correction to a fermion propagator. However, in the RB case shrinking the remaining sub-diagram to a point would generate a non-renormalizable vertex F-F-H-H. These diagrams therefore present potentially genuine models.

So far we have worked from the full set of (topologies and) diagrams, excluding one after the other the non-interesting cases. However, for those remaining 20 diagrams, there is one more subtlety to be discussed: one can write down Lagrangians, which produce, say, only one neutrino mass at tree-level (or 1-loop) level, while the other neutrino mass ${ }^{6}$ (or masses) are generated radiatively. In this case, restrictions on the particle content of the model are determined by the requirements at that lower order. For example, a model with one right-handed neutrino will produce one non-zero neutrino mass at tree-level, while the other neutrino masses are then automatically generated by the genuine 2-loop diagram PTBM-1 (with SM $W^{+}$gauge bosons).

The following additional (but rather trivial) conditions, which finally guarantee that Lagrangians producing the diagrams in figure 4 are genuine 2-loop Lagrangians - in our sense - should therefore be understood as constraints per neutrino generation for which one wants to generate genuine 2-loop masses. Genuiness in this sense requires:

i) Absence of hypercharge zero fermion electroweak singlets or triplets, or hypercharge 2 scalar $\mathrm{SU}(2)$ triplets is required, otherwise the neutrino mass will be determined by tree level type-(I,II,III) seesaw diagrams.

ii) Absence of hypercharge zero scalar $\mathrm{SU}(2)$ singlets or triplets. The presence of these fields allow constructing 1-loop diagrams by (a) making a simple cut in the 2-loop diagram, or (b) (only in case of triplets) allow to construct the 1-loop diagram T-3.

iii) Internal scalars should not have the quantum numbers of the Higgs, otherwise for diagrams CLBZ-1 and 7, PTBM-1,4,5 and RB-1 a 1-loop diagram exists, no matter the position or flow of the Higgs quantum numbers. For the remaining diagrams, internal scalars with quantum numbers as the Higgs are allowed only if they "flow out" of the vertices connecting two fermions, i.e if calculating for $\overline{\nu_{L}^{c}} m_{\nu} \nu_{L}$ the Yukawa

\footnotetext{
${ }^{6}$ Recall, that oscillation data require only two non-zero neutrino masses.
} 


\begin{tabular}{|l|c|c|c|c|c|c|c|}
\hline \multicolumn{7}{|c|}{ PTBM-3 model } \\
\hline FIELDS & $F_{a}$ & $F_{b}$ & $F_{c}$ & $S_{1}$ & $S_{2}$ & $S_{3}$ & $S_{4}$ \\
\hline $\mathrm{SU}(2)_{L}$ & 2 & $(1,3)$ & 2 & 1 & 2 & 2 & 1 \\
$\mathrm{U}(1)_{Y}$ & $q+3$ & 2 & $q+1$ & $q+2$ & $q+3$ & $q+1$ & $q$ \\
\hline
\end{tabular}

Table 2. Quantum number assignment for the new particles appearing in the PTBM-3 model, which is the one possible exception to rule iv. Naming conventions for particles as in figure 10. Strict application of rule iv, would forbid this model. However, for any $q$ different from zero this model has no lower order neutrino mass diagram.

coupling has the structure: $\overline{F_{a}} F_{b} H^{\dagger}$ or $\overline{F_{c}} L H^{\dagger} .{ }^{7}$ Otherwise also for those remaining diagrams a 1-loop diagram will be possible drawing as well.

iv) And, lastly, in order to guarantee absence of 1-loop contributions from T-3, if not already excluded by the previous three conditions, one needs to check the $\mathrm{SU}(2)$ quantum numbers of internal scalars. The quartic vertex in T-3 can be generated [38] by attaching a pair of Higgses to $S_{1} S_{2}$ with $S_{1}=S_{D}$ and $S_{2}=S_{D}$ or $S_{1}=S_{S}$ and $S_{2}=S_{T}$ or $S_{1}=S_{T}$ and $S_{2}=S_{T}$, where $S, D$ and $T$ indicate singlet, doublet and triplet under $\mathrm{SU}(2)$. If any of these combinations appear, the difference in hypercharge of these states must be different from $2 Y_{H}$ in order to forbid T-3. For RB diagrams, this rule applies for $S_{1}$ being a scalar in the inner loop and $S_{2}$ a scalar in the outer loop. Different from all previous conditions, this rule has (exactly) one exception, see table 2.

\subsection{SM gauge quantum numbers}

Due to the large number of diagrams involved, it is desirable to apply a strategy where the quantum number assignments are done mostly at the topology level rather than at the diagrammatic level. Since both the leptons and the Higgs are SU(2) doublets, for these quantum number this turns out to be trivially possible. However, for hypercharge $\left(Y=2\left(Q-T_{3}\right)\right)$ the procedure is more subtle due to the different hypercharges the lepton and Higgs doublets have. This implies that different external lepton-Higgs attachments lead to different hypercharges for the internal fields. So, when discussing hypercharge assignments, in order to avoid a diagrammatic approach we group the different diagrams according to the different external lepton-Higgs structures, which once fixed lead to a unique set of hypercharges for the internal fields.

For all the relevant topologies we will label the internal fields as $X_{i}$ (see figure 7), where $X_{i}$ can be either a scalar or a fermion depending on the specific diagram (figure 4 and 5). For the field $X_{i}$, no matter whether it is a fermion or a scalar, we will use the notation $r$ for the $\mathrm{SU}(2)$ quantum numbers (with $r$ labelling the $\mathrm{SU}(2)$ representation $r=1,2,3$ : singlet, doublet and triplet). Hypercharge of a given field $X_{i}$ will be denoted by $Y_{i}$. In what follows we discuss quantum number assignments for the double-box diagrams CLBZ-i

\footnotetext{
${ }^{7}$ If instead one calculates for $\overline{\nu_{R}} m_{\nu}^{*} \nu_{R}^{c}$, the "flow out" will be defined by the Hermitian conjugate of these couplings.
} 


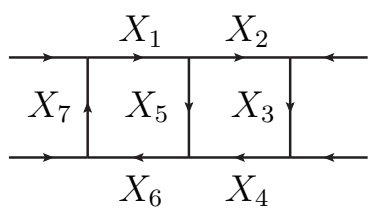

(a)

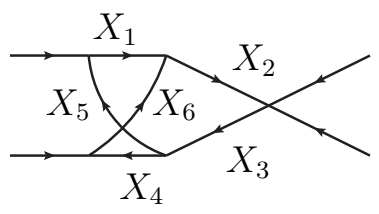

(d)

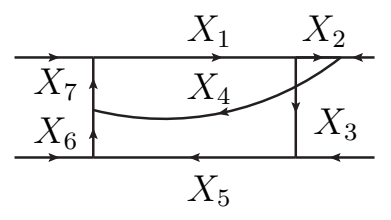

(b)

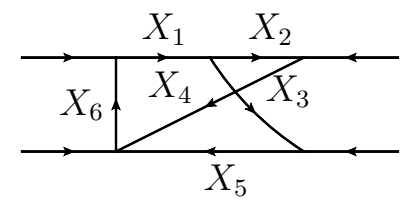

$(e)$

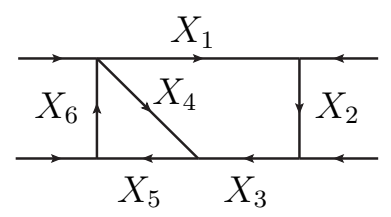

$(c)$

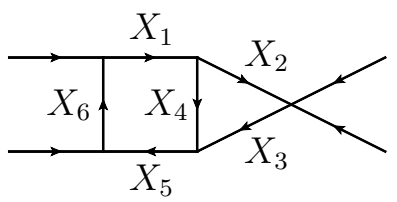

$(f)$

Figure 7. Symbolic internal field assignments for the different renormalizable and genuine two-loop diagrams in figures 4 and $5 . X_{i}$ holds either for fermion or bosons, the specific choice is determined by the diagrams in figures 4 and 5 .

and PTBM-i $(\mathrm{i}=1,2,3)$ in figure 4 and RB-j $(\mathrm{j}=1,2)$ in figure 5. Results for the remaining diagrams are summarized in appendix A. For all possible genuine diagrams we display the possible quantum number assignments in tables.

Quantum numbers for diagrams of type (a) figure 7: we start with $T 2_{1}^{\mathrm{B}}$-based diagrams, as shown in figure 7-(a). SU(2) invariance of the different vertices imply the following constraints:

$$
\begin{array}{ll}
X_{1} \otimes X_{2} \supset \bar{X}_{5}, & X_{1} \otimes X_{7} \supset 2, \\
X_{7} \otimes X_{6} \supset 2, & X_{2} \otimes X_{3} \supset 2, \\
X_{3} \otimes X_{4} \supset 2 . &
\end{array}
$$

This means that fixing the representation of $X_{1,2}$ fixes $X_{5}$. With $X_{1}$ fixed $X_{7}$ is determined too, and this in turn allows settling $X_{6}$. With $X_{2}$ specified, $X_{3}$ can be determined as well and this finally fixes $X_{4}$. The $\mathrm{SU}(2)$ assignment "chain" is then given by: $X_{1,2} \rightarrow X_{5}$; $X_{1} \rightarrow X_{7} \rightarrow X_{6} ; X_{2} \rightarrow X_{3} \rightarrow X_{4}$.

The setup of constraints in (2.14), (2.15) and (2.16) are summarized in table 3, where in addition to the $\mathrm{SU}(2)$ possible quantum number assignments (upper table) we have as well added a table with the different set of possible hypercharge assignments (lower table).

Some words are in order regarding table 3 . The upper table is divided in three subtables delimited by the double vertical lines. The subtable in the left hand side shows the possible $\mathrm{SU}(2)$ charges of the internal fields for $X_{1}$ fixed to be a singlet and for any $X_{2}$ (singlet, doublet and triplet). The following subtables give the $\mathrm{SU}(2)$ charges for $X_{1}$ transforming as a doublet (middle subtable) or as a triplet (right hand side subtable) for any $X_{2}$. Note that there is no relation between the choices for $X_{1}$ and $X_{2}$, e.g. while $X_{1}$ can transform as a triplet $X_{2}$ can do so as a singlet. For fields which admit two $\mathrm{SU}(2)$ charge assignments within a certain row (see e.g. $X_{6}$ and $X_{4}$ for $X_{1,2} \sim 1$ or $X_{6}$ and $X_{3}$ for $X_{1} \sim 1$ and $X_{2} \sim 2$ 


\begin{tabular}{|c|c|c|c|c|c|c|c|c|c|c|c|c|c|c|c|}
\hline \multicolumn{16}{|c|}{$\mathrm{SU}(2)$ quantum numbers } \\
\hline$X_{2} X_{1}$ & \multicolumn{5}{|c|}{1} & \multicolumn{5}{|c|}{2} & \multicolumn{5}{|c|}{3} \\
\hline & $X_{5}$ & $X_{7}$ & $X_{6}$ & $X_{3}$ & $X_{4}$ & $X_{5}$ & $X_{7}$ & $X_{6}$ & $X_{3}$ & $X_{4}$ & $X_{5}$ & $X_{7}$ & $X_{6}$ & $X_{3}$ & $X_{4}$ \\
\hline \multirow{2}{*}{1} & \multirow{2}{*}{1} & \multirow{2}{*}{2} & 1 & \multirow{2}{*}{2} & 1 & \multirow{2}{*}{2} & 1 & \multirow{2}{*}{2} & \multirow{2}{*}{2} & 1 & \multirow{2}{*}{3} & \multirow{2}{*}{2} & 1 & \multirow{2}{*}{2} & 1 \\
\hline & & & 3 & & 3 & & 3 & & & 3 & & & 3 & & 3 \\
\hline \multirow{2}{*}{2} & \multirow{2}{*}{2} & \multirow{2}{*}{2} & 1 & 1 & \multirow{2}{*}{2} & 1 & 1 & \multirow{2}{*}{2} & 1 & \multirow{2}{*}{2} & \multirow{2}{*}{2} & \multirow{2}{*}{2} & 1 & 1 & \multirow{2}{*}{2} \\
\hline & & & 3 & 3 & & 3 & 3 & & 3 & & & & 3 & 3 & \\
\hline \multirow{2}{*}{3} & \multirow{2}{*}{3} & \multirow{2}{*}{2} & 1 & \multirow{2}{*}{2} & 1 & \multirow{2}{*}{2} & 1 & \multirow{2}{*}{2} & \multirow{2}{*}{2} & 1 & 1 & \multirow{2}{*}{2} & 1 & \multirow{2}{*}{2} & 1 \\
\hline & & & 3 & & 3 & & 3 & & & 3 & 3 & & 3 & & 3 \\
\hline
\end{tabular}

\begin{tabular}{|c|c|c|c|c|c|c|c|}
\hline \multicolumn{10}{|c|}{ Hypercharge } \\
\hline$S_{i}$ & $Y_{1}$ & $Y_{2}$ & $Y_{3}$ & $Y_{4}$ & $Y_{5}$ & $Y_{6}$ & $Y_{7}$ \\
\hline$S_{1}$ & $-1+\alpha$ & $1+\beta$ & $\beta$ & $1+\beta$ & $-2+\alpha-\beta$ & $-1+\alpha$ & $\alpha$ \\
\hline$S_{2}$ & $-1+\alpha$ & $-1+\beta$ & $\beta$ & $-1+\beta$ & $\alpha-\beta$ & $-1+\alpha$ & $\alpha$ \\
\hline$S_{3}$ & $-1+\alpha$ & $-1+\beta$ & $\beta$ & $1+\beta$ & $\alpha-\beta$ & $1+\alpha$ & $\alpha$ \\
\hline
\end{tabular}

Table 3. Electroweak quantum numbers for diagrams CLBZ-i, PTBM-i $(i=1,2,3)$ and RB-j $(j=1,2)$ in figure 4 and figure 5. Upper table: $\mathrm{SU}(2)$ representations. Lower table: hypercharge assignments. Fields $X_{i}$ refer to internal fields in the symbolic diagram in figure 7-(a). Symbols $S_{i}$ refer to allowed external lepton-Higgs structures according to figure 8. Hypercharge of field $X_{i}$ is denoted by $Y_{i}$ (see the text for further details). Since the lepton and Higgs doublets are color singlets, color charges can be trivially included.

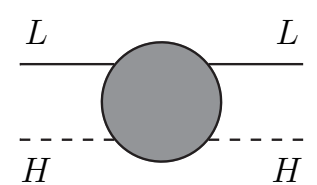

$\left(S_{1}\right)$

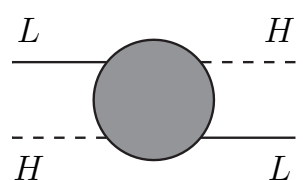

$\left(S_{2}\right)$

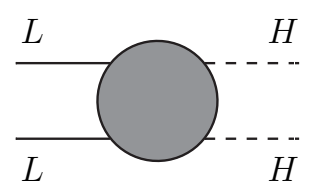

$\left(S_{3}\right)$

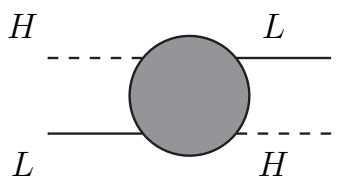

$\left(S_{4}\right)$

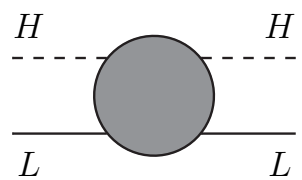

$\left(S_{5}\right)$

Figure 8. Possible external $L H$ structures used to determine the internal fields hypercharges. 
in table 3), a horizontal internal line indicates that no crossed assignments are possible. For example, when $X_{1,2} \sim 1, X_{6}$ can transform as either a singlet or a triplet. If fixed to be a singlet (triplet), $X_{4}$ is fixed univocally to be a singlet (triplet) too. If instead the horizontal internal line is absent, crossing is possible. This is indeed the case for $X_{6}$ and $X_{3}$ when $X_{1} \sim$ 1 and $X_{2} \sim 2$. Fixing $X_{6}$ to be a singlet (triplet) allows $X_{3}$ to be either a singlet or a triplet.

Finally, the lower table shows the different hypercharge assignments derived by taking hypercharge flow according to figure 7, and the possible lepton-Higgs external structures $S_{1}$, $S_{2}$ and $S_{3}$, schematically represented in figure 8 . Note that since the number of internal fields exceed the number of hypercharge conservation constraints (one per each vertex), hypercharge is not univocally fixed. The arbitrariness is encoded in the parameters $\alpha$ and $\beta$.

We do not give explicitly color quantum numbers in our tables. As mentioned above, the inclusion of color is straightforward, since Higgs and lepton doublets are color singlets. This implies that (pairs of) internal particles coupled to either $L$ or $H$ can come only in combinations of $1 \otimes 1,3 \otimes \overline{3}, 6 \otimes \overline{6}$ etc. Moreover, once the colour quantum numbers for internal particles coupled to $L$ or $H$ are chosen, the color quantum numbers of the remaining inner particles are fixed by consistency conditions, derived from $\mathrm{SU}(3)$ rules such as $\overline{3} \otimes \overline{3}=3_{a} \oplus \overline{6}_{s}$ and $3 \otimes \overline{3}=1 \oplus 8$.

\subsubsection{Assigning quantum numbers: some examples}

We now exemplify the use of these results by constructing a couple of models. For that purpose we take diagrams CLBZ-1 and PTBM-1 (see figure 4):

- A CLBZ-1-based model:

starting with CLBZ-1, and comparing with the symbolic diagram in figure 7 it can be seen that: $X_{1,2,5} \rightarrow S_{1,2,5}$ and $X_{3,4,6,7} \rightarrow F_{3,4,6,7}$. Whether the resulting model involves three (four) different scalar (fermion) fields should be determined by their transformation properties, for which table 6 should be used.

Sticking to the case $X_{1,2} \sim 1$, one is left with $X_{5} \sim 1$ and $X_{7} \sim 2$. For $X_{6}$ there are two possible choices, taking $X_{6} \sim 1$ one then has $X_{3} \sim 2$ and univocally $X_{4} \sim 1$. Diagram CLBZ-1 follows a $S_{1}$ lepton-Higgs structure (see figure 8 ), so for hypercharge assignments one has to focus on the $S_{1}$ row in table 6 . Fixing $\alpha=-1$ and $\beta=1$, one gets $Y_{1}=-2, Y_{2}=2, Y_{4}=2, Y_{5}=-4, Y_{6}=-2$. So, the resulting UV completion consist of: one hypercharge +2 scalar singlet and its complex conjugate $\left(S_{2}=S_{1}^{*}\right)$, one hypercharge +4 scalar singlet $\left(S_{5}\right)$, one hypercharge -1 fermion doublet and its conjugate $\left(F_{3}=\bar{F}_{7}\right)$, and one hypercharge -2 fermion singlet and its conjugate $\left(F_{4}=\bar{F}_{6}\right)$. Thus, the fermions can be identified with SM lepton doublets and singlets, and so the UV completion constructed in this way is nothing else but the CLBZ model $[16,26,27]$. Other quantum number choices, as dictated by table 3 , will of course produce variants of the CLBZ model.

- A PTBM-1-based model:

in this case comparing diagram PTBM-1 with that in figure 7-(a) allows the identification: $X_{1,4} \rightarrow S_{1,4}$ and $X_{2,3,5,6,7} \rightarrow F_{2,3,5,6,7}$. For the $\mathrm{SU}(2)$ charges we fix 


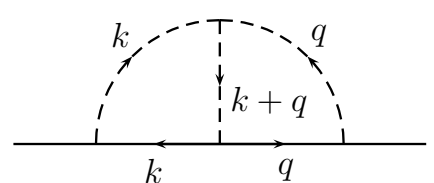

(1)

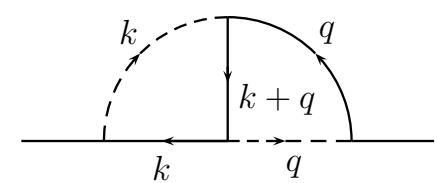

(2)

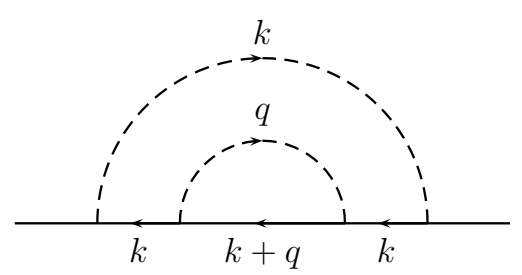

$(3)$

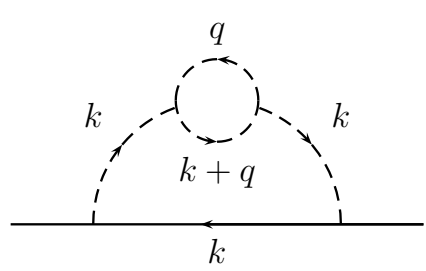

(4)

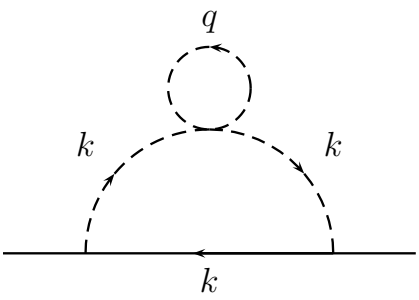

(5)

Figure 9. Diagrams determining the type of integrals one encounters in 2-loop models. Diagrams (1) and (2) in the upper row correspond to CLBZ and PTBM like models, whereas diagrams in the lower row correspond to RB (3) and ISC ((4) and (5)) models. Note that the latter leads only to non-genuine 2-loop models.

them as in the previous example. For hypercharge one has to bear in mind the lepton-Higgs structure, which for this diagram follows $S_{2}$ (see figure 8). Thus, fixing $\alpha=\beta=1 / 3$ one gets the following UV completion: a hypercharge $+1 / 3$ fermion doublet and its copy $\left(F_{3}=F_{7}=F\right)$, one hypercharge $-2 / 3$ fermion singlet and its copy $\left(F_{2}=F_{6}=F^{\prime}\right)$, one vanishing hypercharge fermion singlet $\left(F_{5}=f\right)$ and one hypercharge $-2 / 3$ scalar singlet and its copy $\left(S_{1}=S_{4}\right)$. Assigning non-trivial color charges to these fields: $f \sim 8_{c}, F \sim 3_{c}, F \sim 3_{c}$ and $S_{1}=S_{4} \sim \overline{3}$, one can then identify $F$ with quark $\mathrm{SU}(2)$ doublets while $F^{\prime}$ with quark $\mathrm{SU}(2)$ singlets. The resulting model in that case then matches the model of Angel et al. [60]. Using table 3, further variants can be constructed.

\section{Constructing two-loop models}

Figure 9 shows diagramatically the different classes of integrals that one encounters in the calculation of two-loop models. Diagrams (1) to (3) show the case that can correspond to "genuine" or "true 2-loop" models, while diagrams corresponding to ISC-i and ISC-ii diagrams (diagrams (4) and (5) respectively) always correspond to non-genuine models. We will discuss in the following two examples, one for genuine models (PTBM-3) and one non-geniune model (based on NG-RB-1).

\subsection{Genuine 2-loop models}

In genuine 2-loop models of either CLBZ or PTBM type one encounters two types of integrals: ${ }^{8}$

$$
\mathcal{I}_{a b, \alpha \beta, X}=\frac{1}{(2 \pi)^{8}} \int d^{4} k \int d^{4} q \frac{1}{\left(k^{2}-m_{a}^{2}\right)\left(k^{2}-m_{\alpha}^{2}\right)\left(q^{2}-m_{b}^{2}\right)\left(q^{2}-m_{\beta}^{2}\right)\left[(q+k)^{2}-m_{X}^{2}\right]},
$$

\footnotetext{
${ }^{8}$ In the appendix we give also formulas for RB type diagrams.
} 


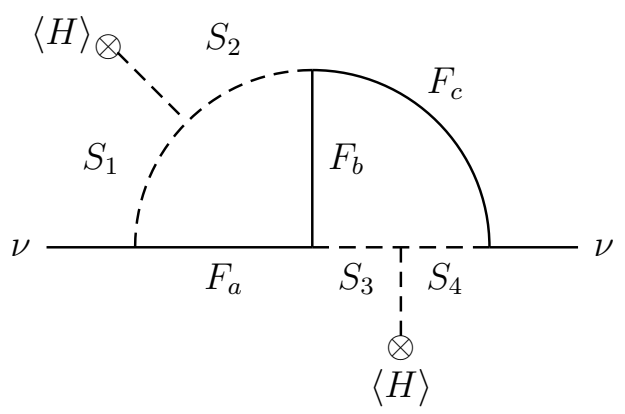

Figure 10. Example for a genuine 2-loop model. The diagram corresponds to PTBM-3 in figure 4 in section 2.4.

$$
\mathcal{I}_{a b, \alpha \beta, X}^{\left\{k^{2}, q^{2},(q+k)^{2}\right\}}=\frac{1}{(2 \pi)^{8}} \int d^{4} k \int d^{4} q \frac{\left\{k^{2}, q^{2},(q+k)^{2}\right\}}{\left(k^{2}-m_{a}^{2}\right)\left(k^{2}-m_{\alpha}^{2}\right)\left(q^{2}-m_{b}^{2}\right)\left(q^{2}-m_{\beta}^{2}\right)\left[(q+k)^{2}-m_{X}^{2}\right]} .
$$

Here, $\left\{k^{2}, q^{2},(q+k)^{2}\right\}$ implies that the numerator could be any of $k^{2}, q^{2}$ or $(q+k)^{2}$, depending on the helicity structure of the underlying Lagrangian, see discussion below. We choose the convention of labelling the fermion masses as $a, b$ and the scalar masses as $\alpha, \beta . X$ is the inner particle that can be either a scalar (CLBZ-type) or fermion (PTBMtype). Note that integral in (3.1) is finite per se, while a finite result for integrals (3.2) requires summation over internal mass eigenstates.

Integrals in (3.1) and (3.2) can be evaluated by rewriting them in terms of a "master integral" (see eq. (C.11) in appendix C). In order to illustrate the way in which this is done, we write down a specific PTBM-3-based model which arises from the diagram shown in figure 10. For all other possible genuine 2-loop models, the procedure follows very closely the one outlined for this particlar example.

The diagram in figure 10 is generated from the following Lagrangian

$$
\mathcal{L}_{\text {int }}=Y_{i a}\left(\overline{L_{i}^{c}} P_{L} S_{1}\right) \cdot F_{a}^{c}+Y_{c j}\left(\overline{F_{c}} P_{L} L_{j}\right) \cdot S_{4}+h_{a b} \overline{F_{a}^{c}} \cdot\left(F_{b}^{c} S_{3}^{\dagger}\right)+h_{b c}\left(\overline{F_{b}^{c}} F_{c}\right) \cdot S_{2}^{\dagger}+\text { H.c. },
$$

and scalar potential terms

$$
V \supset \mu_{34} S_{4}^{\dagger} \cdot\left(S_{3} H\right)+\mu_{12} S_{2} \cdot\left(S_{1}^{\dagger} H\right)+\text { H.c. }+\sum_{x=1}^{4} m_{S_{x}}^{2}\left|S_{x}\right|^{2},
$$

where the parenthesis indicate $\mathrm{SU}(2)$ index contractions. The particle content of the resulting model and its SM transformation properties are displayed in table 4. In addition, the fermions can have vectorlike mass terms, namely:

$$
\mathcal{L}_{M}=\sum_{A=a, b, c} m_{F_{A}} \overline{F_{A}} F_{A} .
$$

Coupling $\mu_{34}$ in (3.4) induces mixing between the $Q=3 / 2$ scalars, while $\mu_{12}$ mixing between the $Q=1 / 2$ states. The mass matrices for these states then reads

$$
M_{S^{Q=3 / 2}}^{2}=\left(\begin{array}{cc}
m_{S_{3}}^{2} & \mu_{34} v \\
\mu_{34} v & m_{S_{4}}^{2}
\end{array}\right), \quad M_{S^{Q=1 / 2}}^{2}=\left(\begin{array}{cc}
m_{S_{1}}^{2} & \mu_{12} v \\
\mu_{12} v & m_{S_{2}}^{2}
\end{array}\right) .
$$




\begin{tabular}{|l|c|c|c|c|c|c|c|}
\hline \multicolumn{7}{|c|}{ PTBM-3 model } \\
\hline FIELDS & $F_{a}$ & $F_{b}$ & $F_{c}$ & $S_{1}$ & $S_{2}$ & $S_{3}$ & $S_{4}$ \\
\hline $\mathrm{SU}(2)_{L}$ & 1 & 2 & 2 & 2 & 1 & 2 & 1 \\
$\mathrm{U}(1)_{Y}$ & 1 & 5 & -4 & 2 & 1 & -4 & -3 \\
\hline
\end{tabular}

Table 4. Quantum number assignment for the new particles appearing in the diagram shown in figure 10. For simplicity, all states are assumed to be color singlets.

Assuming the mixing parameters to be real, these matrices are diagonalized by $2 \times 2$ rotation matrices:

$$
R_{Q}=\left(\begin{array}{cc}
\cos \theta_{Q} & \sin \theta_{Q} \\
-\sin \theta_{Q} & \cos \theta_{Q}
\end{array}\right)
$$

with the rotation angles given by:

$$
\tan 2 \theta_{Q=3 / 2}=\frac{2 \mu_{34} v}{m_{S_{3}}^{2}-m_{S_{4}}^{2}}, \quad \tan 2 \theta_{Q=1 / 2}=\frac{2 \mu_{12} v}{m_{S_{1}}^{2}-m_{S_{2}}^{2}} .
$$

Rotating the interactions in (3.3) and (3.4) to the scalar mass eigenstate basis, one can then calculate the full neutrino mass matrix.

The chiral structures appearing in the diagram are determined by the different chiral projectors $\left(P_{L}\right.$ or $\left.P_{R}\right)$ entering in each of the Yukawa vertices involved. Since chirality of external vertices (those where the SM neutrinos enter) is fixed by the neutrino chirality, the number of possibilities is determined by the different chiral structures of internal Yukawa vertices. For PTBM models there are three chiral structures: internal vertices with $P_{L}-P_{L}$, $P_{R}-P_{L}$ or $P_{R}-P_{R}$ stuctures. The (internal) combination $P_{L}-P_{L}$ leads to integrals of type eq. (3.1), while the other two possibilities project out integrals of type eq. (3.2). The full final result reads:

$\mathcal{M}_{\nu}=\frac{1}{4\left(16 \pi^{2}\right)^{2}}\left(Y_{i a} Y_{c j}+Y_{j a} Y_{c i}\right) h_{a b} h_{b c} \sin 2 \theta_{Q=3 / 2} \sin 2 \theta_{Q=1 / 2} \sum_{A=1}^{4} \sum_{\alpha, \beta}(-1)^{\alpha}(-1)^{\beta} F_{a c, \alpha \beta, b}^{(A)}$,

with the different dimensionful functions $F_{a c, \alpha \beta, b}^{(A)}$, determined by

$$
\begin{aligned}
& F_{a b, \alpha \beta, b}^{(1)}=\frac{m_{F_{a}} m_{F_{c}}}{m_{F_{b}}} \times \pi^{-4} \hat{\mathcal{I}}_{a c, \alpha \beta}, \\
& F_{a b, \alpha \beta, b}^{(2)}=\left(m_{F_{a}}+m_{F_{b}}+m_{F_{c}}\right) \times \pi^{-4} \hat{\mathcal{I}}_{a c, \alpha \beta}^{\left[(k+q)^{2}\right]}, \\
& F_{a c, \alpha \beta, b}^{(3)}=-\left(m_{F_{a}}+m_{F_{b}}\right) \times \pi^{-4} \hat{\mathcal{I}}_{a c, \alpha \beta}^{\left(k^{2}\right)}, \\
& F_{a c, \alpha \beta, b}^{(4)}=-\left(m_{F_{b}}+m_{F_{c}}\right) \times \pi^{-4} \hat{\mathcal{I}}_{a c, \alpha \beta}^{\left(q^{2}\right)},
\end{aligned}
$$

With the aid of eqs. (C.12)-(C.15) in appendix C, one can then express these functions in terms of the "master" function $\hat{g}(s, t)$ (see eq. (C.23)).

Figure 11 shows some examples of calculated neutrino masses for different choices of input parameters. This calculation does not take into account any flavour structure in 

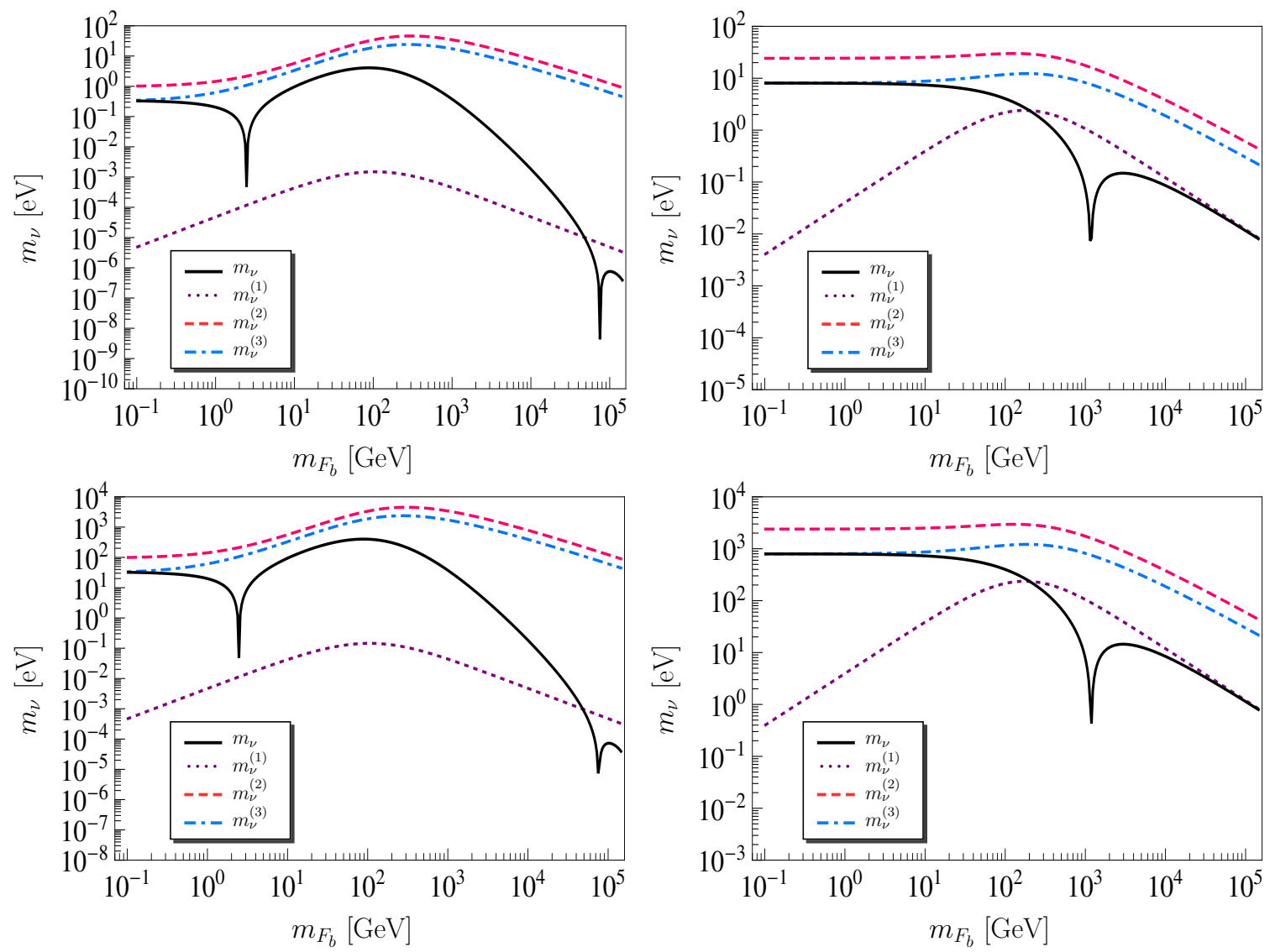

Figure 11. Examples of calculated neutrino mass as function of $m_{F_{b}}$ for four different sets of input parameters, see text. The full line shows the total $m_{\nu}$, the other lines the individual contributions $m_{\nu}^{(i)}$, determined by the functions $F_{a b, \alpha \beta, b}^{(i)}$ (see eqs. (3.10)-(3.12)) and the common global factor in (3.9). The plots are for Yukawa couplings equal to 1 and thus show that neutrino masses of the correct order of magnitude can be obtained easily in this model.

the indices of Yukawa couplings, i.e. $Y_{i a}=Y_{a}$ etc, and puts the values of all Yukawas $Y_{a}=Y_{c}=h_{a b}=h_{b c}=1$. The numerical values of $m_{\nu}$ should therefore be understood as the typical scale of neutrino mass and not as an exact prediction for the three light neutrino mass eigenvalues, see the discussion on flavour fits below. Also, note, that while the numerical values shown for $m_{\nu}$ are a bit too large compared to, say, the atmospheric neutrino scale, $\sqrt{\Delta\left(m_{\text {Atm }}^{2}\right)} \simeq 0.05 \mathrm{eV}$, this could be easily adjusted for using smaller values for the Yukawas.

The plots then show $m_{\nu}$ as a function of $m_{F_{b}}$ for scalar mass parameters $m_{S_{1}}^{2}=$ $100^{2} \mathrm{GeV}^{2}$ and $m_{S_{2}}^{2}=m_{S_{1}}^{2}+\Delta m^{2}$ (with $\Delta m^{2}=\mu v$ ), for two different, fixed $\Delta m^{2}=$ $24.6 \mathrm{GeV}^{2}$ (upper row) and $246 \mathrm{GeV}^{2}$ (lower row) and two different values of $m_{F}=m_{F_{a}}=$ $m_{F_{c}}$ : to the left $1 \mathrm{GeV}$ and to the right $100 \mathrm{GeV}$. The black (full) line shows the total $m_{\nu}$, the other lines show the different contributions $m_{\nu}^{(i)}, i=1,2,3$ individually (determined by the functions in (3.10)-(3.12) and the common global factor in (3.9)). Note, that $m_{\nu}^{(4)}$ is numerically equal to $m_{\nu}^{(3)}$, while $m_{\nu}^{(2)}<0$ and we plot the absolute value. Usually the contribution 
from $m_{\nu}^{(2)}-m_{\nu}^{(3)}$ dominates the neutrino mass for small and moderate values of $m_{F_{b}}$, but at large values of $m_{F_{b}}, m_{\nu}^{(2)}$ and $m_{\nu}^{(3)}+m_{\nu}^{(4)}$ tend to cancel each other, such that the only remaining contribution comes from $m_{\nu}^{(1)}$. In the plots there are some points for $m_{F_{b}}$, for which the different contributions can actually exactly cancel each other. Note also, that for $m_{F_{b}} \rightarrow$ $\infty, m_{\nu}$ goes to zero, as expected. Obviously, as these plots demonstrate, neutrino masses of the correct order of magnitude can be achieved for a wide range of input parameters.

We close this section with a brief discussion on neutrino flavour fits. Any model, aiming at explaining neutrino oscillation data, must of course not only reproduce the overall neutrino mass scale, but also have sufficient freedom to fit the two neutrino mass squared differences and the three neutrino angles. Our numerical examples have been done with only one non-zero neutrino mass, fits to all data can nevertheless easily be done. The actual form of the fit, however, depends on the number of copies of new fermions and scalars present in the model under consideration. For exactly one copy of new states, both fermions and scalar, eq. (3.9) has rank-2. This implies that one can fit hierarchical neutrino spectra (both normal and inverted), but not degenerate neutrinos. With more copies of scalars or fermions, also degenerate neutrinos can be fitted. In this case, the simplest way to proceed is via a fit analogous to the Casas-Ibarra parametrization for the seesaw (type-I/III) [85]. The authors of [60] have spelled out this procedure for two copies of internal scalars and one vector of Yukawas, i.e. their case is also rank-2. One can devise in a completely analogous way the fit for three-fermion or there-scalar models, simply adapting the formulas from [85], so we will not discuss this in further detail here.

\subsection{Non-genuine but finite 2-loop models}

As discussed at length in the previous sections, some CLBZ, PTBM or RB diagrams will not correspond to genuine 2-loop models. However, models generating such kind of diagrams might still be interesting constructions in the following sense: consider, for example, a model with some new fermions in which, invoking a non-Abelian flavor symmetry, the direct coupling of the new fermions with the standard model Higgs is forbidden. The flavour symmetry is then broken at some large, unspecified scale and upon integrating out some heavy fields, an effective fermion-fermion-Higgs vertex is generated at 1-loop order. Such a construction would allow to understand, at least in principle, why that particular coupling is small compared to all others, simply due to the $1 /\left(16 \pi^{2}\right)$ suppression from the loop. An example of this approach is the $d=7 \mathrm{RB}$ model of [77], but the very same idea could, of course, be applied to any of our non-genuine $d=5$ diagrams.

In all such cases one can carry out the calculation either by solving the full 2-loop integral or by first calculating that particular vertex at 1-loop order and then doing a 1loop calculation for the neutrino mass using this effective vertex in the second step. We will call the former the "full" or "2-loop" calculation, while we call the second approach "2-step" calculation in the following. The two calculations should, of course, lead to the same numerical result (only) in the limit where there is a certain hierarchy of masses for the particles in the loop. In this subsection, we will discuss one particular example of such a model, based on the RB diagram NG-RB-1, see figure 12, in some detail. The treatment of all other "finite" but non-genuine models is very similar. Here, we are mainly interested in 


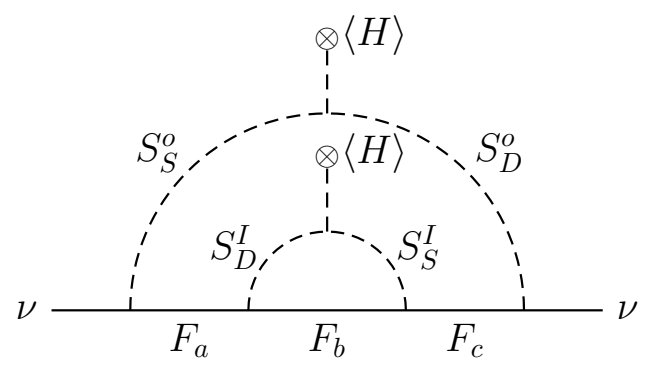

Figure 12. Example for a "finite" RB diagram. The diagram corresponds to NG-RB-1 in figure 17 in appendix A.

\begin{tabular}{|c|c|c|c|c|c|c|c|}
\hline \multicolumn{10}{|c|}{ NG-RB-1 } \\
\hline FIELDS & $F_{a}$ & $F_{b}$ & $F_{c}$ & $S_{S}^{O}$ & $S_{D}^{O}$ & $S_{S}^{I}$ & $S_{D}^{I}$ \\
\hline $\mathrm{SU}(2)_{L}$ & 2 & 1 & 1 & 1 & 2 & 1 & 2 \\
$\mathrm{U}(1)_{Y}$ & 1 & 0 & -2 & 2 & 1 & -2 & 1 \\
\hline
\end{tabular}

Table 5. Quantum number assignment for new particles appearing in the diagram shown in figure 12. All states are assumed to be color singlets for simplicity.

demonstrating the numerical agreement between full and 2-step calculation and therefore will not work out the details of a suitable flavor symmetry model. We again refer to [77] for an example for the $(d=7) \mathrm{RB}$ diagram based on the discrete symmetry $T_{7}$ and to [68] for another example based on a variant of the ISC-i diagram using the symmetry $\mathbb{Z}_{2} \times \mathbb{Z}_{2}^{\prime}$.

The diagram in figure 12 can be generated from the following interaction Yukawa Lagrangian:

$$
\mathcal{L}_{\text {int }}=Y_{i a}\left(\bar{L}_{i}^{c} P_{L} F_{a}^{c}\right) \cdot S_{O}^{S}+Y_{c i} \bar{F}_{c} \cdot\left(S_{O}^{D, \dagger} P_{L} L_{i}\right)+h_{a b}\left(\bar{F}_{a}^{c} S_{I}^{D, \dagger}\right) P_{R} F_{b}+h_{b c} \bar{F}_{b}^{c} P_{R} F_{c} S_{I}^{S}+\text { H.c. } .
$$

This fixes the SM quantum numbers as shown in table 5. The scalars appearing in the inner and outer loops, denoted by $S^{I}$ and $S^{O}$ respectively, have the same SM quantum numbers and thus could be the same particles. For generality, however, and since in the ultra-violet completion they could transform differently under the flavour group, we will treat them as independent states.

The scalar potential of the model contains the following terms:

$$
V \supset \mu_{O}\left(S_{D}^{O} H\right) . S_{S}^{O, \dagger}+\mu_{I}\left(S_{D}^{I} H\right) . S_{S}^{I}+\text { H.c. }+\sum_{\substack{x=D, S \\ y=O, I}}\left(m_{x}^{y}\right)^{2}\left|S_{x}^{y}\right|^{2} .
$$

We add then the following three fermion mass terms:

$$
\mathcal{L}_{M}=m_{F_{a}} F_{a} \bar{F}_{a}+m_{F_{b}} F_{b} \bar{F}_{b}^{c}+m_{F_{c}} F_{c} \bar{F}_{c} .
$$

Only $F_{b}$ can have a Majorana mass, as indicated by the charge conjugation " $C$ " in eq. (3.16), but $F_{a}$ and $F_{c}$ can have vector-like fermion mass terms. ${ }^{9}$

\footnotetext{
${ }^{9}$ We note that, the presence of the Majorana fermion, $F_{b}$, together with the scalar $S^{D}$ allows, in principle,
} 
The mass matrices for the inner and outer scalars can be written as

$$
M_{S^{k}}^{2}=\left(\begin{array}{cc}
m_{D^{k}}^{2} & \mu_{k} v \\
\mu_{k} v & m_{S^{k}}^{2}
\end{array}\right)
$$

with $k=I, O$. This matrix can be diagonalized, as in the example in the previous section, by a simple rotation matrix with the angle given as:

$$
\tan \left(2 \theta_{k}\right)=\frac{2 \mu_{k} v}{m_{D^{k}}^{2}-m_{S^{k}}^{2}} .
$$

The expression for the neutrino mass is given as

$$
\begin{aligned}
\left(\Delta m_{\nu}\right)_{i j}= & \frac{1}{4\left(16 \pi^{2}\right)^{2}}\left(Y_{i a} Y_{j c}+Y_{j a} Y_{i c}\right) h_{a b} h_{b c} \sin \left(2 \theta_{O}\right) \sin \left(2 \theta_{I}\right) m_{F_{b}} \\
& \times \sum_{\alpha, \beta=1,2}(-1)^{\alpha}(-1)^{\beta} \pi^{-4} \hat{\mathcal{I}}_{a c, \alpha \beta, b}^{\left(k^{2}\right) \mathrm{RB}}
\end{aligned}
$$

with $\hat{\mathcal{I}}_{a c, \alpha \beta}^{\left(k^{2}\right) \mathrm{RB}}$ given by eq. (C.17) in appendix C.

Now consider the 2 -step calculation. The inner loop can be evaluated as

$$
\Delta_{m}=\frac{1}{2} h_{a b} h_{b c} m_{F_{b}} \sin \left(2 \theta_{I}\right) \mathcal{I}_{t_{x_{1}}, t_{x_{2}}}
$$

with $^{10}$

$$
\mathcal{I}_{t_{x_{1}}, t_{x_{2}}}=\frac{1}{(2 \pi)^{4}} \int d^{4} q \frac{1}{\left(q^{2}-t_{x_{1}}\right)\left(q^{2}-t_{x_{2}}\right)\left(q^{2}-1\right)} .
$$

The solution to eq. (3.21) gives the well-known function

$$
\mathcal{I}_{t_{x_{1}}, t_{x_{2}}}=\frac{i}{16 \pi^{2}} \times\left\{\frac{t_{x_{2}}}{t_{x_{2}}-1} \ln \left(t_{x_{2}}\right)-\frac{t_{x_{1}}}{t_{x_{1}}-1} \ln \left(t_{x_{1}}\right)\right\} .
$$

$\Delta_{m}$ gives an entry to the mass matrix of the fermions $F_{a}$ and $F_{c}$ :

$$
\mathcal{M}_{F_{a} F_{c}}=\left(\begin{array}{cc}
m_{F_{a}} & \Delta_{m} \\
\Delta_{m} & m_{F_{c}}
\end{array}\right)
$$

Diagonalizing this mass matrix gives two eigenvalues $m_{F_{1}}$ and $m_{F_{2}}$, which can be used in the calculation of the outer loop, which has the same form than the inner loop just calculated. This results in:

$$
\left(\Delta m_{\nu}\right)_{i j}^{2-\text { step }}=\frac{1}{2}\left(Y_{i a} Y_{j c}+Y_{j a} Y_{i c}\right) h_{a b} h_{b c} \sum_{\alpha=1,2} m_{F_{\alpha}} V_{\alpha 1} V_{\alpha 2} \sin \left(2 \theta_{O}\right) \mathcal{I}_{t_{y_{1}}, t_{y_{2}}}
$$

where $V_{i j}$ is the matrix which diagonalizes eq. (3.23).

to construct a 1-loop diagram for the neutrino mass, once the coefficient of $\lambda_{5}\left(S^{D, \dagger} H\right)\left(S^{D, \dagger} H\right)$ is nonzero [39]. This coupling must be forbidden by some symmetry in order to make the diagram NG-RB-1 the leading contribution to the neutrino mass.

${ }^{10} \mathcal{I}_{t_{x_{1}}, t_{x_{2}}}$ is essentially the difference of two Passarino-Veltman $B_{0}(0, s, t)$ functions, see appendix. We prefer to write it this way to make the contact with the notation in [39]. 

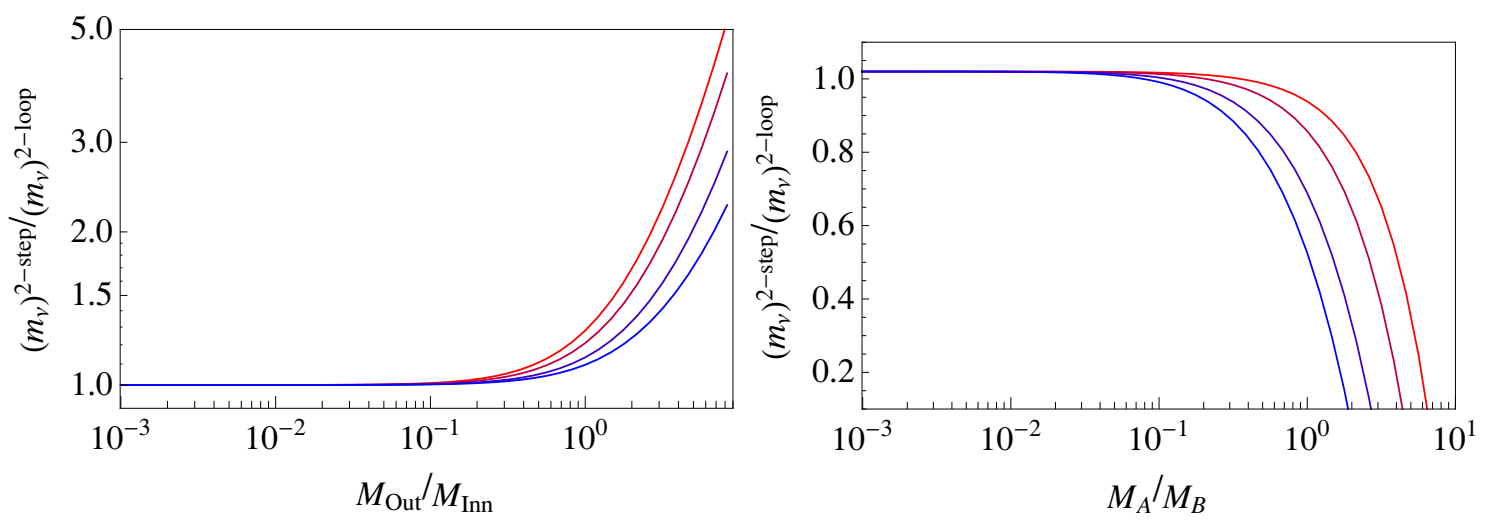

Figure 13. Comparison of the full 2-loop calculation to the approximate "2-step" calculation. The plots show the ratio of the approximate calculation to the full calculation. To the left: outer fermions $F_{a}$ and $F_{c}$ have negligibly small masses, $m_{S_{D}^{O}}=m_{S_{S}^{O}}=M_{O u t}$ and $m_{F_{b}}=m_{S_{S}^{I}}=M_{I n n}$. The four different lines are (from top to bottom) $m_{S_{D}^{I}} / M_{I n n}=1,2,5$ and 10. To the right $m_{S_{D}^{O}}=$ $m_{S_{S}^{O}}=M_{O u t}$ and $m_{S_{S}^{I}}=m_{S_{D}^{I}}=10 M_{O u t}$, as a function of $m_{F_{a}} / m_{F_{b}}$ for four different values (from top to bottom) of $m_{F_{c}} / m_{F_{a}}=0.1,0.2,0.5$ and 1 .

Figure 13 shows a comparison of the neutrino mass calculated with eq. (3.19) (full 2loop result) and eq. (3.24) (2-step result) for different combinations of internal masses. We show the ratio of the two calculations, thus all coupling constants cancel and need not to be specified. The calculation is for one neutrino mass only and not meant to be a complete fit to all neutrino data. The plot on the left shows the result for negligibly small outer fermion masses, varying the (common) mass of the inner-loop particles, keeping the masses of the outer scalars constant. The plot on the right shows the result for fixed values of the scalar masses, but varying the ratio of inner to outer fermion mass. In both cases, it is clear that if there is a hierarchy in the masses of the particles in the inner loop with respect to the masses in the outer loop, then the two calculations agree very well. Comparison of the plot on the right to the plot on the left demonstrates that especially the value of the ratio of the fermion masses is important: fermion $F_{b}$ should be heavier than the outer fermions, otherwise the 2-step calculation starts to fail.

We close of this discussion with one more comment. Eq. (3.14) specifies that the vertex connecting $F_{a}$ and $F_{b}$ has a projector $P_{R}$. However, we have given a vector-like mass term to these fermions and vector-like fermions can couple, in prinicple, with both chiralities. A model, in which the other projector $P_{L}$ also appears, however, will produce terms proportional to $\left(m_{F_{a}}+m_{F_{c}}\right) q . k$ and, different from the case discussed here, can not be calculated in the "2-step" method described here, which relies on picking the internal fermion mass $m_{F_{b}}$.

\section{Conclusions}

Using a diagrammatic approach we have systematically studied the $d=5$ Weinberg operator at the 2-loop order. Out of the large number of possible diagrams the majority are just corrections to lower order diagrams. We have shown that the relevant 2-loop models can be classified as follows: (A) Class-I models, which only involve genuine diagrams, 
i.e. diagrams for which the absence of lower order diagrams is assured. Interestingly, we have found that class-I models implicate only variants of the CLBZ (Cheng-Li-Babu-Zee) models [16, 26, 27], PTBM (Petcov-Toshev-Babu-Ma) models [41, 42] and RB (rainbow) models. (B) Class-II models, which involve non-genuine but finite 2-loop diagrams. Diagrams belonging to this class correspond to 1-loop diagrams that contain a 1-loop generated vertex, and are variations of just five different diagrams which we have dubbed: NG-CLBZ (non-genuine CLBZ), NG-PTBM (non-genuine PTBM), NG-RB (non-genuine RB), ISC-i and ISC-ii (internal scalar correction type i and ii).

We provided the full list of class-I diagrams in figure 4 and 5. This list combined with our results for the internal fields SM quantum number assignments (summarized in tables 3,6-10), allows the construction of novel 2-loop neutrino mass models, something that we have exemplified in section 2.5.1 and, in more details in section 3. We have given as well the full list of non-genuine but finite 2-loop diagrams in figures 16-19. This list enables the construction of novel 2-loop models where the smallness of certain coupling can be, in principle, understood as due to its 1-loop radiative origin. Also, the "tools" needed for numerical calculations have been collected in appendix C.

In summary, we have identified the possible 2-loop neutrino mass models arising from the $d=5$ Weinberg operator. Our findings can be understood as a guide for the construction of 2-loop neutrino mass models, which arguably might serve for several purposes, e.g: systematic study of neutrino mass model signals at the LHC (testing the origin of neutrino masses at the LHC, as has been pointed out at the 1-loop level e.g. in [86-88], and at the 2-loop level in [89]) or systematic construction of common frameworks for neutrino masses and dark matter (in the same vein it has been done for the 1-loop case [90]).

\section{Acknowledgments}

We thank Renato Fonseca for discussions and for pointing out an inconsistency in the first version of the draft. DAS wants to thank Yasaman Farzan for conversations and useful comments. DAS is supported by a "Chargè de Recherches" contract funded by the Belgian FNRS agency. MH acknowldeges support by Spanish MICINN grants FPA2011-22975, MULTIDARK CSD2009-00064, the Generalitat Valenciana grant PrometeoII/2014/084 and FCT grant EXPL/FIS-NUC/0460/2013.

\section{A Non-renormalizable topologies and finite non-genuine diagrams}

In this appendix, we present in figure 14 the list of renormalizable topologies involving non-genuine but finite 2-loop diagrams. For completeness in figure 15 we display as well the full set of non-renormalizable topologies we have found. As we have already mentioned, 2-loop non-genuine but finite diagrams arise from 1-loop diagrams where one of the internal vertices is generated at the 1-loop order. 2-loop finite non-genuine diagrams can therefore be classified according to the 1-loop diagram from which they originate. Figures 16-19 show the different finite non-genuine diagrams classified according to this scheme. 

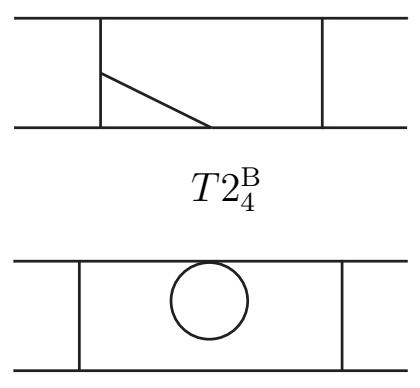

$T 2_{7}^{\mathrm{B}}$

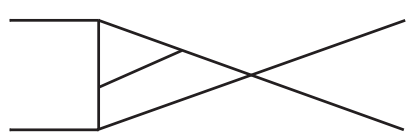

$T 2_{4}^{\mathrm{T}}$

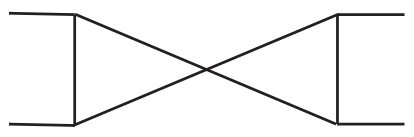

$T 2_{7}^{\mathrm{T}}$

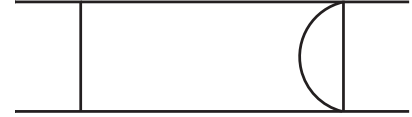

$T 2_{5}^{\mathrm{B}}$

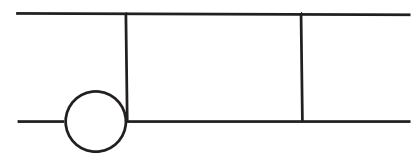

$T 2_{8}^{\mathrm{B}}$

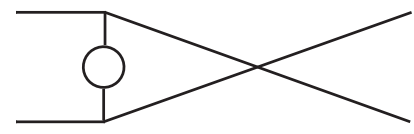

$T 2_{5}^{\mathrm{T}}$

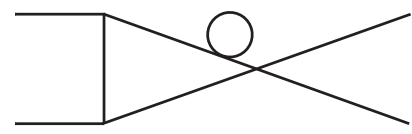

$T 2_{8}^{\mathrm{T}}$

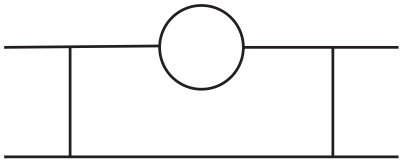

$T 2_{6}^{\mathrm{B}}$

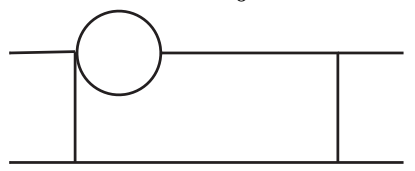

$T 2_{9}^{\mathrm{B}}$

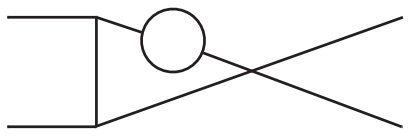

$T 2_{6}^{\mathrm{T}}$

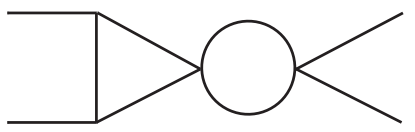

$T 2_{9}^{\mathrm{T}}$

Figure 14. 1PI two-loop topologies leading to non-genuine finite or infinite diagrams. Topologies $T 2_{4,6}^{B}$ belong to set $(6,0)$, topologies $T_{5}^{B}$ and $T_{8,9}^{T}$ to $(2,2)$, while the remaining to the $(4,1)$ set. Further details can be found in section 2.2 .

\section{B Quantum numbers}

In this appendix, we give tables from which the SM quantum numbers of genuine diagrams CLBZ-i and PTBM-i $(\mathrm{i}=4,5,6, \ldots)$ as well as RB- $\mathrm{j}(\mathrm{j}=3,4)$ can be determined. The tables obey the same conventions as table 3 , i.e. symbols $S_{i}$ refer to allowed external leptonHiggs structures according to figure 8, and hypercharge of field $X_{i}$ is denoted by $Y_{i}$. Their utilization requires as well using figure 7 , as already discussed and exemplified in section 2.5.

We start with table 6 , which provides the possible quantum number assignments for genuine diagrams CLBZ-i and PTBM-i (with $\mathrm{i}=4,5,6)$ in figure 4 and RB-3 in figure 5 . Table 7, instead, gives the possible assignments for genuine diagrams CLBZ-7 and CLBZ8, whereas tables 8 and 9 for genuine diagrams CLBZ-9 and CLBZ-10, respectively. Finally, table 10 gives the assignments for diagram RB-4 in figure 5. We note again that due to the lepton and Higgs doublets being color singlets, color charges for internal fields can be straightforwardly included, and so we do not list them.

We point out that in order to construct compact tables, we have written in some cases two possibilities for $\mathrm{SU}(2)$ assignments of particles. Usually this would lead to 8 possible combinations, for which, however, not all are allowed. Exceptions are those where vertices are (obviously) forbidden by $\mathrm{SU}(2)$ invariance or do not yield the Weinberg operator. 


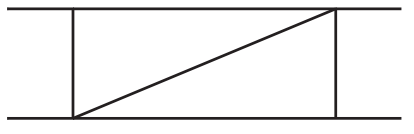

$T 2_{1}^{\mathrm{NR}}$

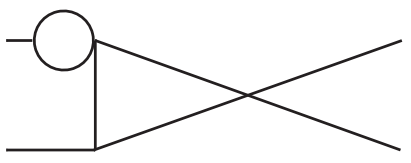

$T 2_{4}^{\mathrm{NR}}$

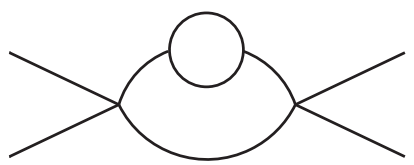

$T 2_{7}^{\mathrm{NR}}$

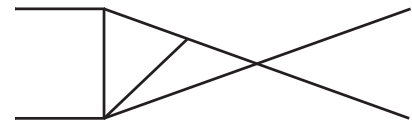

$T 2_{2}^{\mathrm{NR}}$

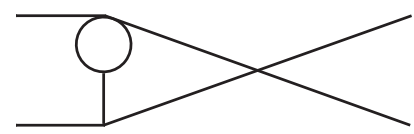

$T 2_{5}^{\mathrm{NR}}$

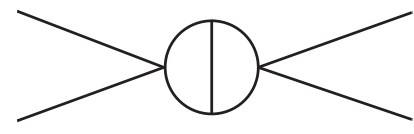

$T 2_{8}^{\mathrm{NR}}$

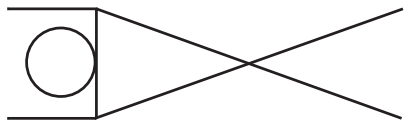

$T 2_{3}^{\mathrm{NR}}$

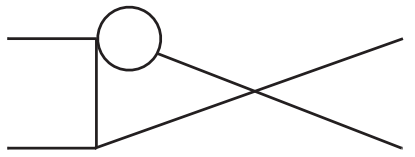

$T 2_{6}^{\mathrm{NR}}$

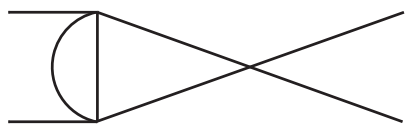

$T 2_{9}^{\mathrm{NR}}$

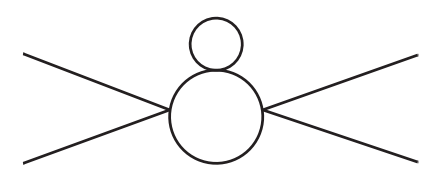

$T 2_{10}^{\mathrm{NR}}$

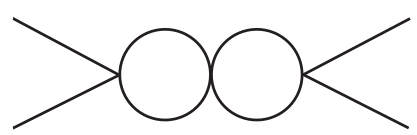

$T 2_{11}^{\mathrm{NR}}$

Figure 15. 1PI two-loop topologies not satisfying the renormalizability condition. The first eight topologies belong to $(2,2)$ set while the last three to the set $(0,3)$ set. Further details can be found in section 2.2 .

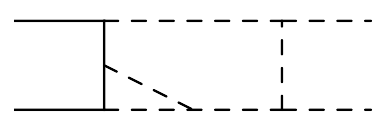

NG-CLBZ-1

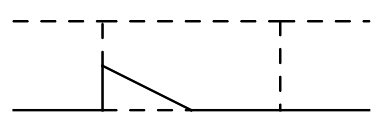

NG-PTBM-1

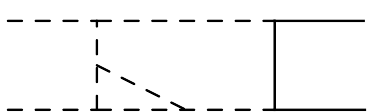

ISC-i-1

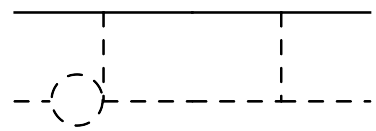

ISC-ii-1

Figure 16. Non-genuine and finite two-loop diagrams which correspond to the one-loop generation of one of the couplings entering in the one-loop diagram T1-i (see figure 1).

Therefore, when using tables 6-10, one should bear in mind that neither triple vertices with combinations of representations 1-1-3 (or any of its permutations) nor combinations which lead to (an effective) quartic vertex $H H-1-1$ are allowed.

\section{Useful formulas for 2-loop calculations}

The integrals appearing in the 2-loop diagrams have been evaluated several times in the literature. We follow [60, 91], both of which are based on [92]. We repeat here only the basic definitions and final results, for more details see [60, 91, 92]. 


\begin{tabular}{|c|c|c|c|c|c|c|c|c|c|c|c|c|c|c|c|}
\hline \multicolumn{16}{|c|}{$\mathrm{SU}(2)$ quantum numbers } \\
\hline$X_{5} X_{1}$ & \multicolumn{5}{|c|}{1} & \multicolumn{5}{|c|}{2} & \multicolumn{5}{|c|}{3} \\
\hline & $X_{3}$ & $X_{2}$ & $X_{4}$ & $X_{6}$ & $X_{7}$ & $X_{3}$ & $X_{2}$ & $X_{4}$ & $X_{6}$ & $X_{7}$ & $X_{3}$ & $X_{2}$ & $X_{4}$ & $X_{6}$ & $X_{7}$ \\
\hline 1 & 2 & 2 & $\begin{array}{l}1 \\
3\end{array}$ & 2 & 2 & 2 & $\begin{array}{l}1 \\
3\end{array}$ & 2 & 2 & $\begin{array}{l}1 \\
3\end{array}$ & 2 & 2 & $\begin{array}{l}1 \\
3\end{array}$ & 2 & 2 \\
\hline \multirow{2}{*}{2} & 3 & 1 & \multirow{2}{*}{2} & 1 & \multirow{2}{*}{2} & 1 & \multirow{2}{*}{2} & 1 & 1 & 1 & 1 & 3 & \multirow{2}{*}{2} & 1 & \multirow{2}{*}{2} \\
\hline & 1 & 3 & & 3 & & 3 & & 3 & 3 & 3 & 3 & 1 & & 3 & \\
\hline 3 & 2 & 2 & $\begin{array}{l}1 \\
3\end{array}$ & 2 & 2 & 2 & $\begin{array}{l}1 \\
3\end{array}$ & 2 & 2 & $\begin{array}{l}1 \\
3\end{array}$ & 2 & 2 & $\begin{array}{l}1 \\
3\end{array}$ & 2 & 2 \\
\hline
\end{tabular}

\begin{tabular}{|c|c|c|c|c|c|c|c|}
\hline \multicolumn{7}{|c|}{ Hypercharge } \\
\hline$S_{i}$ & $Y_{1}$ & $Y_{2}$ & $Y_{3}$ & $Y_{4}$ & $Y_{5}$ & $Y_{6}$ & $Y_{7}$ \\
\hline$S_{1}$ & $-1+\alpha$ & $-1+\alpha-\beta$ & $\beta$ & $-2+\alpha-\beta$ & $1+\beta$ & $2+\beta$ & $\alpha$ \\
\hline$S_{2}$ & $-1+\alpha$ & $-1+\alpha-\beta$ & $\beta$ & $\alpha-\beta$ & $-1+\beta$ & $\beta$ & $\alpha$ \\
\hline$S_{3}$ & $-1+\alpha$ & $-1+\alpha-\beta$ & $\beta$ & $\alpha-\beta$ & $1+\beta$ & $\beta$ & $\alpha$ \\
\hline$S_{5}$ & $1+\alpha$ & $1+\alpha-\beta$ & $\beta$ & $2+\alpha-\beta$ & $-1+\beta$ & $-2+\beta$ & $\alpha$ \\
\hline
\end{tabular}

Table 6. Electroweak quantum numbers for diagrams CLBZ-i and PTBM-i $(i=4,5,6)$ in figure 4 and RB-3 in figure 5. Upper table: $\mathrm{SU}(2)$ representations. Lower table: hypercharge assignments. Fields $X_{i}$ refer to internal fields in the symbolic diagram in figure $7-(b)$.

\begin{tabular}{|c|c|c|c|c|c|c|c|c|c|c|c|c|}
\hline \multicolumn{13}{|c|}{ SU(2) quantum numbers } \\
\hline$\widehat{X_{6}} X_{1}$ & \multicolumn{4}{|c|}{1} & \multicolumn{4}{|c|}{2} & \multicolumn{4}{|c|}{3} \\
\hline & $X_{2}$ & $X_{3}$ & $X_{4}$ & $X_{5}$ & $X_{2}$ & $X_{3}$ & $X_{4}$ & $X_{5}$ & $X_{2}$ & $X_{3}$ & $X_{4}$ & $X_{5}$ \\
\hline 1 & 2 & $\begin{array}{l}1 \\
3\end{array}$ & 2 & 2 & $\begin{array}{l}1 \\
3\end{array}$ & 2 & $\begin{array}{l}1 \\
3\end{array}$ & 2 & 2 & $\begin{array}{l}1 \\
3\end{array}$ & 2 & 2 \\
\hline 2 & 2 & $\begin{array}{l}1 \\
3\end{array}$ & 2 & 2 & $\begin{array}{l}1 \\
3\end{array}$ & 2 & 2 & $\begin{array}{l}1 \\
3\end{array}$ & 2 & $\begin{array}{l}1 \\
3\end{array}$ & $\begin{array}{l}1 \\
3\end{array}$ & $\begin{array}{l}1 \\
3\end{array}$ \\
\hline 3 & 2 & $\begin{array}{l}1 \\
3\end{array}$ & 2 & 2 & $\begin{array}{l}1 \\
3\end{array}$ & 2 & $\begin{array}{l}1 \\
3\end{array}$ & 2 & 2 & $\begin{array}{l}1 \\
3\end{array}$ & 2 & 2 \\
\hline
\end{tabular}

\begin{tabular}{|c|c|c|c|c|c|c|}
\hline \multicolumn{7}{|c|}{ Hypercharge } \\
\hline$S_{i}$ & $Y_{1}$ & $Y_{2}$ & $Y_{3}$ & $Y_{4}$ & $Y_{5}$ & $Y_{6}$ \\
\hline$S_{4}$ & $1+\beta$ & $\beta$ & $1+\beta$ & $\alpha-\beta$ & $1+\alpha$ & $\alpha$ \\
\hline$S_{5}$ & $-1+\beta$ & $\beta$ & $-1+\beta$ & $2+\alpha-\beta$ & $1+\alpha$ & $\alpha$ \\
\hline
\end{tabular}

Table 7. Electroweak quantum numbers for diagrams CLBZ-7 and CLBZ-8 in figure 4. Upper table: $\mathrm{SU}(2)$ representations. Lower table: hypercharge assignments. Fields $X_{i}$ refer to internal fields in the symbolic diagram in figure 7-(c). 


\begin{tabular}{|c|c|c|c|c|c|c|c|c|c|c|c|c|}
\hline \multicolumn{13}{|c|}{ SU(2) quantum numbers } \\
\hline$X_{4} X_{1}$ & \multicolumn{4}{|c|}{1} & \multicolumn{4}{|c|}{2} & \multicolumn{4}{|c|}{3} \\
\hline & $X_{5}$ & $X_{6}$ & $X_{2}$ & $X_{3}$ & $X_{5}$ & $X_{6}$ & $X_{2}$ & $X_{3}$ & $X_{5}$ & $X_{6}$ & $X_{2}$ & $X_{3}$ \\
\hline \multirow{2}{*}{1} & \multirow{2}{*}{2} & \multirow{2}{*}{2} & \multirow{2}{*}{2} & \multirow{2}{*}{2} & 1 & \multirow{2}{*}{2} & 1 & 1 & \multirow{2}{*}{2} & \multirow{2}{*}{2} & \multirow{2}{*}{2} & \multirow{2}{*}{2} \\
\hline & & & & & 3 & & 3 & 3 & & & & \\
\hline \multirow{2}{*}{2} & \multirow{2}{*}{2} & 1 & 1 & 1 & 1 & 1 & \multirow{2}{*}{2} & \multirow{2}{*}{2} & \multirow{2}{*}{2} & 1 & 3 & 1 \\
\hline & & 3 & 3 & 3 & 3 & 3 & & & & 3 & 1 & 3 \\
\hline \multirow{2}{*}{3} & \multirow{2}{*}{2} & \multirow{2}{*}{2} & \multirow{2}{*}{2} & \multirow{2}{*}{2} & 1 & \multirow{2}{*}{2} & 1 & 3 & \multirow{2}{*}{2} & \multirow{2}{*}{2} & \multirow{2}{*}{2} & \multirow{2}{*}{2} \\
\hline & & & & & 3 & & 3 & 1 & & & & \\
\hline & \multicolumn{11}{|c|}{ Hypercharge } & \\
\hline$S_{i}$ & \multicolumn{3}{|c|}{$Y_{1}$} & $Y_{2}$ & $Y$ & 3 & $Y_{4}$ & $Y_{5}$ & \multicolumn{3}{|c|}{$Y_{6}$} & \\
\hline$S_{3}$ & \multicolumn{3}{|c|}{$-2-\alpha-\beta$} & $-2+$ & $\beta$ & \multicolumn{2}{|c|}{$1+\alpha$} & $\alpha$ & \multicolumn{3}{|c|}{$-1-\alpha+\beta$} & \\
\hline
\end{tabular}

Table 8. Electroweak quantum numbers for diagrams CLBZ-9 in figure 4. Upper table: SU(2) representations. Lower table: hypercharge assignments. Fields $X_{i}$ refer to internal fields in the symbolic diagram in figure $7-(d)$.

\begin{tabular}{|c|c|c|c|c|c|c|c|c|c|c|c|c|}
\hline \multicolumn{13}{|c|}{$\mathrm{SU}(2)$ quantum numbers } \\
\hline$X_{3} X_{1}$ & \multicolumn{4}{|c|}{1} & \multicolumn{4}{|c|}{2} & \multicolumn{4}{|c|}{3} \\
\hline & $X_{2}$ & $X_{4}$ & $X_{5}$ & $X_{6}$ & $X_{2}$ & $X_{4}$ & $X_{5}$ & $X_{6}$ & $X_{2}$ & $X_{4}$ & $X_{5}$ & $X_{6}$ \\
\hline 1 & 2 & 2 & $\begin{array}{l}1 \\
3\end{array}$ & 2 & 2 & $\begin{array}{l}1 \\
3\end{array}$ & 2 & $\begin{array}{l}1 \\
3\end{array}$ & 2 & 2 & $\begin{array}{l}1 \\
3\end{array}$ & 2 \\
\hline \multirow[t]{2}{*}{2} & 1 & 1 & \multirow[t]{2}{*}{2} & \multirow[t]{2}{*}{2} & 1 & \multirow[t]{2}{*}{2} & \multirow{2}{*}{$\begin{array}{l}1 \\
3\end{array}$} & \multirow{2}{*}{$\begin{array}{l}1 \\
3\end{array}$} & 1 & 3 & \multirow[t]{2}{*}{2} & \multirow{2}{*}{2} \\
\hline & 3 & 3 & & & 3 & & & & 3 & 1 & & \\
\hline 3 & 2 & 2 & $\begin{array}{l}1 \\
3\end{array}$ & 2 & 2 & $\begin{array}{l}1 \\
3\end{array}$ & 2 & $\begin{array}{l}1 \\
3\end{array}$ & 2 & 2 & $\begin{array}{l}1 \\
3\end{array}$ & 2 \\
\hline
\end{tabular}

\begin{tabular}{|c|c|c|c|c|c|c|}
\hline \multicolumn{7}{|c|}{ Hypercharge } \\
\hline$S_{i}$ & $Y_{1}$ & $Y_{2}$ & $Y_{3}$ & $Y_{4}$ & $Y_{5}$ & $Y_{6}$ \\
\hline$S_{1}$ & $-1+\alpha$ & $1+\beta$ & $\beta$ & $-2+\alpha-\beta$ & $-1+\alpha-\beta$ & $\alpha$ \\
\hline
\end{tabular}

Table 9. Electroweak quantum numbers for diagram CLBZ-10 in figure 4. Upper table: SU(2) representations. Lower table: hypercharge assignments. Fields $X_{i}$ refer to internal fields in the symbolic diagram in figure $7-(e)$. 


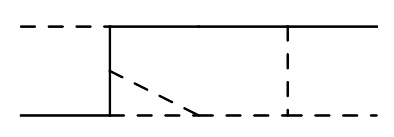

NG-CLBZ-2

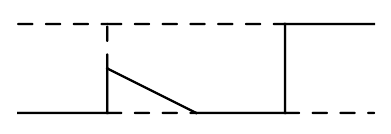

NG-PTBM-2

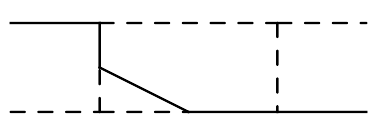

NG-RB-1

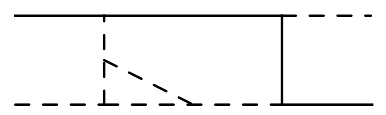

ISC-i-2

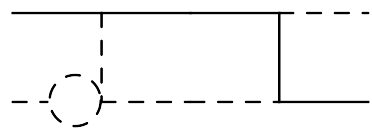

ISC-ii-2

Figure 17. Non-genuine and finite two-loop diagrams which correspond to the one-loop generation of one of the couplings entering in the one-loop diagram T1-ii (see figure 1).

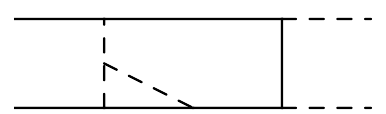

NG-CLBZ-3

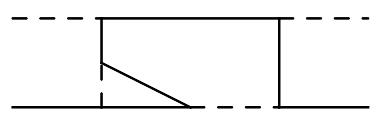

NG-PTBM-3

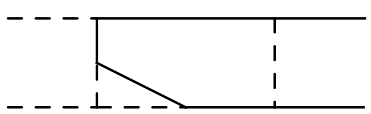

NG-RB-2

Figure 18. Non-genuine and finite two-loop diagrams which correspond to the one-loop generation of one of the couplings entering in the one-loop diagram T1-iii (see figure 1).

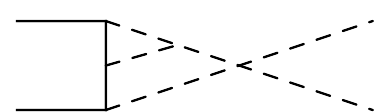

NG-CLBZ-4

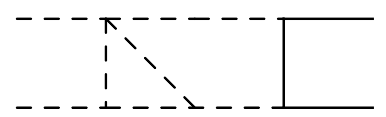

ISC-i-4

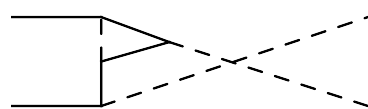

NG-PTBM-4

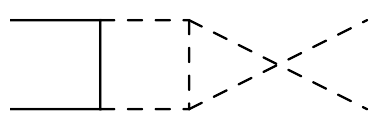

ISC-i-5

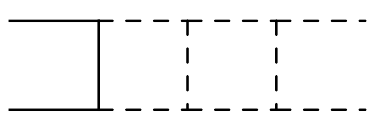

ISC-i-3

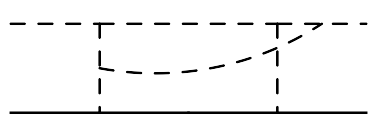

ISC-i-6

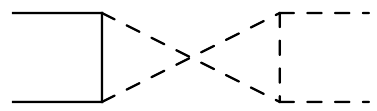

ISC-ii-3

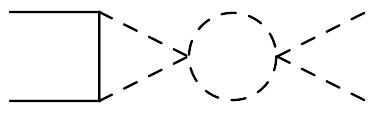

ISC-ii-4

Figure 19. Non-genuine and finite two-loop diagrams which correspond to the one-loop generation of one of the couplings entering in the one-loop diagram T-3 (see figure 1).

As a starting point, define [92]

$$
\begin{aligned}
& \left(M_{11}, \cdots, M_{1 n_{1}}\left|M_{21}, \cdots, M_{2 n_{2}}\right| M_{31}, \cdots, M_{3 n_{3}}\right) \\
& \quad=\int d^{n} p \int d^{n} q \Pi_{i=1}^{n_{1}} \Pi_{j=1}^{n_{2}} \Pi_{k=1}^{n_{3}} \frac{1}{\left(p^{2}+M_{1 i}^{2}\right)} \frac{1}{\left(q^{2}+M_{2 j}^{2}\right)} \frac{1}{\left[(p+q)^{2}+M_{3 k}^{2}\right]} .
\end{aligned}
$$

Here, $n$ is the number of dimensions. In the case of infinite integrals one has to carefully evaluate all terms for $n=4+\epsilon$ before taking the limit $\epsilon \rightarrow 0$. Since we are interested only in models with finite integrals, we will not write out terms containing poles in $1 / \epsilon$. 


\begin{tabular}{|c|c|c|c|c|c|c|c|c|c|c|c|c|}
\hline \multicolumn{13}{|c|}{$\mathrm{SU}(2)$ quantum numbers } \\
\hline$X_{3} X_{1}$ & \multicolumn{4}{|c|}{1} & \multicolumn{4}{|c|}{2} & \multicolumn{4}{|c|}{3} \\
\hline & $X_{2}$ & $X_{4}$ & $X_{5}$ & $X_{6}$ & $X_{2}$ & $X_{4}$ & $X_{5}$ & $X_{6}$ & $X_{2}$ & $X_{4}$ & $X_{5}$ & $X_{6}$ \\
\hline \multirow{2}{*}{1} & \multirow{2}{*}{3} & \multirow{2}{*}{3} & \multirow{2}{*}{3} & \multirow{2}{*}{2} & \multirow{2}{*}{3} & \multirow{2}{*}{2} & \multirow{2}{*}{2} & 1 & \multirow{2}{*}{3} & 1 & 1 & \multirow{2}{*}{2} \\
\hline & & & & & & & & 3 & & 3 & 3 & \\
\hline 2 & 2 & 2 & $\begin{array}{l}1 \\
3\end{array}$ & 2 & 2 & $\begin{array}{l}1 \\
3\end{array}$ & 2 & $\begin{array}{l}1 \\
3\end{array}$ & 2 & 2 & $\begin{array}{l}1 \\
3\end{array}$ & 2 \\
\hline \multirow{2}{*}{3} & 1 & 1 & 1 & \multirow{2}{*}{2} & 1 & \multirow{2}{*}{2} & \multirow{2}{*}{2} & 1 & 1 & 1 & 1 & \multirow{2}{*}{2} \\
\hline & 3 & 3 & 3 & & 3 & & & 3 & 3 & 3 & 3 & \\
\hline
\end{tabular}

\begin{tabular}{|c|c|c|c|c|c|c|}
\hline \multicolumn{7}{|c|}{ Hypercharge } \\
\hline$S_{i}$ & $Y_{1}$ & $Y_{2}$ & $Y_{3}$ & $Y_{4}$ & $Y_{5}$ & $Y_{6}$ \\
\hline$S_{3}$ & $\alpha$ & $\alpha-\beta$ & $2+\alpha-\beta$ & $\beta$ & $2+\alpha$ & $1+\alpha$ \\
\hline
\end{tabular}

Table 10. Electroweak quantum numbers for diagram RB-4 in figure 5. Upper table: SU(2) representations. Lower table: hypercharge assignments. Fields $X_{i}$ refer to internal fields in the symbolic diagram in figure $7-(f)$.

Using partial fractions, one can rewrite integrals of the form of eq. (C.1) as sums over integrals with fewer denominators:

$$
\left(m, m_{0}\left|m_{1}\right| m_{2}\right)=\frac{1}{m^{2}-m_{0}^{2}}\left\{\left(m_{0}\left|m_{1}\right| m_{2}\right)-\left(m\left|m_{1}\right| m_{2}\right)\right\} .
$$

Similarly, integrals with three denominators can be recombined into less divergent ones, using [92]

$$
\left(m_{0}\left|m_{1}\right| m_{2}\right)=\frac{1}{3-n}\left\{m_{0}^{2}\left(2 m_{0}\left|m_{1}\right| m_{2}\right)+m_{1}^{2}\left(2 m_{1}\left|m_{0}\right| m_{2}\right)+m_{2}^{2}\left(2 m_{2}\left|m_{0}\right| m_{1}\right)\right\} .
$$

Here, $\left(2 m\left|m_{i}\right| m_{j}\right)$ is a short-hand for $\left(m, m\left|m_{i}\right| m_{j}\right)$. The " $p^{2}$ decomposition" is another relation which proves to be useful for calculating integrals with momentum-dependent numerators, namely

$$
\frac{p^{2}}{\left(p^{2}-m_{1}^{2}\right)\left(p^{2}-m_{2}^{2}\right)}=\frac{1}{\left(p^{2}-m_{1}^{2}\right)}+\frac{m_{2}^{2}}{\left(p^{2}-m_{1}^{2}\right)\left(p^{2}-m_{2}^{2}\right)} .
$$

Using only eq. (C.2) results in expressions [60] which are more compact than those given in [91, 92], which make repeated use of both, eq. (C.2) and eq. (C.3).

Also, for numerical evaluation, it is useful to define the final expressions in terms of dimensionless quantities. By convention we scale all masses appearing in the integrals with respect to the "innermost" scalar/fermion mass. This implies rescaling the momenta, and for $\mathcal{I}_{a b, \alpha \beta, X}$ factoring out this overall mass squared. We thus write

$$
\mathcal{I}_{a b, \alpha \beta, X}=\frac{1}{(2 \pi)^{8}} \frac{1}{m_{X}^{2}} \hat{\mathcal{I}}_{a b, \alpha \beta},
$$




$$
\mathcal{I}_{a b, \alpha \beta}^{\left\{k^{2}, q^{2},(q+k)^{2}\right\}}=\frac{1}{(2 \pi)^{8}} \hat{\mathcal{I}}_{a b, \alpha \beta}^{\left\{k^{2}, q^{2},(q+k)^{2}\right\}}
$$

with

$$
\begin{aligned}
\hat{\mathcal{I}}_{a b, \alpha \beta} & =\int d^{4} k \int d^{4} q \frac{1}{\left(k^{2}-r_{a}\right)\left(k^{2}-t_{\alpha}\right)\left(q^{2}-r_{b}\right)\left(q^{2}-t_{\beta}\right)\left([q+k]^{2}-1\right)}, \\
\hat{\mathcal{I}}_{a b, \alpha \beta}^{\left\{k^{2}, q^{2},(q+k)^{2}\right\}} & =\int d^{4} k \int d^{4} q \frac{\left\{k^{2}, q^{2},(q+k)^{2}\right\}}{\left(k^{2}-r_{a}\right)\left(k^{2}-t_{\alpha}\right)\left(q^{2}-r_{b}\right)\left(q^{2}-t_{\beta}\right)\left([q+k]^{2}-1\right)},
\end{aligned}
$$

for CLBZ and PTBM models, while

$$
\begin{aligned}
\hat{\mathcal{I}}_{a b, \alpha \beta}^{\mathrm{RB}} & =\int d^{4} k \int d^{4} q \frac{1}{\left(k^{2}-r_{a}\right)\left(k^{2}-r_{b}\right)\left(k^{2}-t_{\beta}\right)\left(q^{2}-t_{\alpha}\right)\left([q+k]^{2}-1\right)}, \\
\hat{\mathcal{I}}_{a b, \alpha \beta}^{\left\{k^{2}, q^{2},(q+k)^{2}\right\} \mathrm{RB}} & =\int d^{4} k \int d^{4} q \frac{\left\{k^{2}, q^{2},(q+k)^{2}\right\}}{\left(k^{2}-r_{a}\right)\left(k^{2}-r_{b}\right)\left(k^{2}-t_{\beta}\right)\left(q^{2}-t_{\alpha}\right)\left([q+k]^{2}-1\right)},
\end{aligned}
$$

for RB models. Here $\left\{k^{2}, q^{2},(q+k)^{2}\right\}$ stands for $k^{2}, q^{2}$ or $(k+q)^{2}, r_{a}=\left(m_{F_{a}} / m_{X}\right)^{2}$ and $t_{\alpha}=\left(m_{S_{\alpha}} / m_{X}\right)^{2}$. The "strategy" then for calculating these integrals consists of reducing them to "master integral" form:

$$
I(s, t)=\mu^{\epsilon} \int d^{n} k \int d^{n} q \frac{1}{\left(k^{2}-s\right)\left(q^{2}-t\right)\left[(k+q)^{2}-1\right]} .
$$

This integral, which involves an infinite and a finite piece, has been calculated in [60] (see below). Thus, with the aid of eqs. (C.2) and (C.4), the calculation of integral in (C.7) results in $[60]$

$$
\pi^{-4} \hat{\mathcal{I}}_{a b, \alpha \beta}=\frac{1}{\left(t_{\alpha}-r_{a}\right)\left(t_{\beta}-r_{b}\right)}\left\{-\hat{g}\left(t_{\alpha}, t_{\beta}\right)+\hat{g}\left(r_{a}, t_{\beta}\right)+\hat{g}\left(t_{\alpha}, r_{b}\right)-\hat{g}\left(r_{a}, r_{b}\right)\right\},
$$

while the result for integrals in (C.8) reads

$$
\begin{aligned}
\pi^{-4} \hat{\mathcal{I}}_{a b, \alpha \beta}^{\left(k^{2}\right)}= & \left\{\frac{1}{t_{\beta}-r_{b}}\left[-\hat{g}\left(r_{a}, t_{\beta}\right)+\hat{g}\left(r_{a}, r_{b}\right)\right]\right. \\
& \left.+\frac{t_{\alpha}}{\left(t_{\alpha}-r_{a}\right)\left(t_{\beta}-r_{b}\right)}\left[-\hat{g}\left(t_{\alpha}, t_{\beta}\right)+\hat{g}\left(t_{\alpha}, r_{b}\right)+\hat{g}\left(r_{a}, t_{\beta}\right)-\hat{g}\left(r_{a}, r_{b}\right)\right]\right\}, \\
\pi^{-4} \hat{\mathcal{I}}_{a b, \alpha \beta}^{\left(q^{2}\right)}= & \left\{\frac{1}{t_{\alpha}-r_{a}}\left[-\hat{g}\left(t_{\alpha}, r_{b}\right)+\hat{g}\left(r_{a}, r_{b}\right)\right]\right. \\
& \left.+\frac{t_{\beta}}{\left(t_{\alpha}-r_{a}\right)\left(t_{\beta}-r_{b}\right)}\left[-\hat{g}\left(t_{\alpha}, t_{\beta}\right)+\hat{g}\left(t_{\alpha}, r_{b}\right)+\hat{g}\left(r_{a}, t_{\beta}\right)-\hat{g}\left(r_{a}, r_{b}\right)\right]\right\}, \\
\pi^{-4} \hat{\mathcal{I}}_{a b, \alpha \beta}^{\left\{(k+q)^{2}\right\}}= & \left\{\widehat{B}_{0}^{\prime}\left(0, r_{a}, t_{\alpha}\right) \widehat{B}_{0}^{\prime}\left(0, r_{b}, t_{\beta}\right)+\frac{\left.-\hat{g}\left(t_{\alpha}, t_{\beta}\right)+\hat{g}\left(t_{\alpha}, r_{b}\right)+\hat{g}\left(r_{a}, t_{\beta}\right)-\hat{g}\left(r_{a}, r_{b}\right)\right\} .}{\left(t_{\alpha}-r_{a}\right)\left(t_{\beta}-r_{b}\right)}\right\}
\end{aligned}
$$

Calculation of the integrals in (C.9) and (C.10) gives, instead, the following results:

$$
\begin{aligned}
\pi^{4} \hat{\mathcal{I}}_{a b, \alpha \beta}^{\mathrm{RB}}= & \frac{1}{t_{\beta}-r_{a}}\left\{\frac{1}{t_{\beta}-r_{b}}\left[-\hat{g}\left(t_{\beta}, t_{\alpha}\right)+\hat{g}\left(r_{b}, t_{\alpha}\right)\right]-\frac{1}{r_{b}-r_{a}}\left[-\hat{g}\left(r_{b}, t_{\alpha}\right)+\hat{g}\left(r_{a}, t_{\alpha}\right)\right]\right\}, \\
\pi^{4} \hat{\mathcal{I}}_{a b, \alpha \beta}^{\left(k^{2}\right) \mathrm{RB}}= & \frac{1}{r_{a}-t_{\beta}}\left\{-\hat{g}\left(r_{a}, t_{\alpha}\right)+\hat{g}\left(t_{\beta}, t_{\alpha}\right)\right. \\
& \left.+\frac{r_{b}}{r_{a}-r_{b}}\left[-\hat{g}\left(r_{a}, t_{\alpha}\right)+\hat{g}\left(r_{b}, t_{\alpha}\right)\right]-\frac{r_{b}}{t_{\beta}-r_{b}}\left[-\hat{g}\left(t_{\beta}, t_{\alpha}\right)+\hat{g}\left(r_{b}, t_{\alpha}\right)\right]\right\}, \\
\pi^{4} \hat{\mathcal{I}}_{a b, \alpha \beta}^{\left(q^{2}\right) \mathrm{RB}}= & \frac{t_{\alpha}}{r_{a}-r_{b}}\left\{\frac{1}{r_{a}-t_{\beta}}\left[-\hat{g}\left(r_{a}, t_{\alpha}\right)+\hat{g}\left(t_{\beta}, t_{\alpha}\right)\right]-\frac{1}{r_{b}-t_{\beta}}\left[-\hat{g}\left(r_{b}, t_{\alpha}\right)+\hat{g}\left(t_{\beta}, t_{\alpha}\right)\right]\right\},
\end{aligned}
$$




$$
\begin{aligned}
\pi^{4} \hat{\mathcal{I}}_{a b, \alpha \beta}^{\left\{(k+q)^{2}\right\} \mathrm{RB}}= & \frac{t_{1}-t_{2}}{r_{a}-t_{\beta}}\left[\hat{B}_{0}^{\prime}\left(0, r_{a}, r_{b}\right)-\hat{B}_{0}^{\prime}\left(0, t_{\beta}, r_{b}\right)\right] \hat{B}_{0}^{\prime}\left(0, t_{1}, t_{2}\right) \\
& +\frac{1}{t_{\beta}-r_{a}}\left\{\frac{1}{t_{\beta}-r_{b}}\left[-\hat{g}\left(t_{\beta}, t_{\alpha}\right)+\hat{g}\left(r_{b}, t_{\alpha}\right)\right]-\frac{1}{r_{b}-r_{a}}\left[-\hat{g}\left(r_{b}, t_{\alpha}\right)+\hat{g}\left(r_{a}, t_{\alpha}\right)\right]\right\} .
\end{aligned}
$$

Here, $\hat{g}(s, t)$ is the solution to (C.11), while $\widehat{B}_{0}^{\prime}(0, x, y)$ is given as follows. The well-known one-loop scalar Passarino-Veltman function $B_{0}[93]$ in the vanishing external momentum limit $(p \rightarrow 0)$ reads:

$$
B_{0}(0, s, t)=\int \frac{d^{4} k}{(2 \pi)^{4}} \frac{1}{\left(k^{2}-s\right)\left(k^{2}-t\right)} .
$$

Defining

$$
B_{0}(0, s, t)=\frac{1}{(2 \pi)^{4}} \widehat{B}_{0}(0, s, t),
$$

the finite part of the $\widehat{B}_{0}^{\prime}(0, s, t)$ function can be written according to

$$
\widehat{B}_{0}(0, s, t)=-\pi^{2} i\left(\frac{s \log s-t \log t}{s-t}\right)=\pi^{2} \widehat{B}_{0}^{\prime}(0, s, t),
$$

whereas the finite piece for $\hat{g}(s, t)$ reads:

$$
\begin{aligned}
\hat{g}(s, t)= & \frac{s}{2} \ln s \ln t+\sum_{ \pm} \pm \frac{s(1-s)+3 s t+2(1-t) x_{ \pm}}{2 \omega} \\
& \times\left[\operatorname{Li}_{2}\left(\frac{x_{ \pm}}{x_{ \pm}-s}\right)-\operatorname{Li}_{2}\left(\frac{x_{ \pm}-s}{x_{ \pm}}\right)+\operatorname{Li}_{2}\left(\frac{t-1}{x_{ \pm}}\right)-\operatorname{Li}_{2}\left(\frac{t-1}{x_{ \pm}-s}\right)\right]
\end{aligned}
$$

with the standard di-logarithm

$$
\operatorname{Li}_{2}(x)=-\int_{0}^{x} \frac{\ln (1-y)}{y} d y
$$

and

$$
x_{ \pm}=\frac{1}{2}(-1+s+t \pm \omega) \quad \omega=\sqrt{1+s^{2}+t^{2}-2(s+t+s t)} .
$$

In both cases, $B_{0}$ and $\hat{g}(s, t)$, we have giving expressions only for their finite pieces. The reason is that for the CLBZ or PTBM integral (C.15), we have found that the divergent piece in the first term cancels upon summation over the different contributions. Cancellation of the divergent piece in $\hat{g}(s, t)$, in eqs. (C.12)-(C.15), occurs as well when summing of the the different contributions. For RB integrals, cancellation of divergences proceeds differently: the divergent term from (C.19) cancels with the divergent piece from (C.18). Thus, always rendering finite results for genuine diagrams, as of course it has to be.

Below we repeat the solution for $\hat{\mathcal{I}}_{i j, \alpha \beta}$ in the convention of [91]. This solution rewrites eq. (C.11) into a less divergent expression using eq. (C.3). Introducing dimensionless arguments as before, one finds

$$
\begin{aligned}
\hat{\mathcal{I}}_{i j, \alpha \beta}=\frac{\pi^{4}}{\left(t_{\alpha}-r_{i}\right)\left(t_{\beta}-r_{j}\right)}\left\{r_{i}\left[f\left(t_{\beta} / r_{i}, 1 / r_{i}\right)-f\left(r_{j} / r_{i}, 1 / r_{i}\right)\right]\right. \\
+r_{j}\left[f\left(t_{\alpha} / r_{j}, 1 / r_{j}\right)-f\left(r_{i} / r_{j}, 1 / r_{j}\right)\right]
\end{aligned}
$$




$$
\begin{aligned}
& +t_{\alpha}\left[f\left(r_{j} / t_{\alpha}, 1 / t_{\alpha}\right)-f\left(t_{\beta} / t_{\alpha}, 1 / t_{\alpha}\right)\right] \\
& +t_{\beta}\left[f\left(r_{i} / t_{\beta}, 1 / t_{\beta}\right)-f\left(t_{\alpha} / t_{\beta}, 1 / t_{\beta}\right)\right] \\
& \left.+\left[f\left(t_{\alpha}, r_{j}\right)-f\left(r_{i}, r_{j}\right)-f\left(t_{\alpha}, t_{\beta}\right)+f\left(r_{i}, t_{\beta}\right)\right]\right\} .
\end{aligned}
$$

Here,

$$
\begin{aligned}
f(a, b)=-\frac{1}{2} \ln a \ln b-\frac{1}{2}\left(\frac{a+b-1}{\kappa}\right) & \left\{\operatorname{Li}_{2}\left(\frac{-x_{2}}{y_{1}}\right)+\operatorname{Li}_{2}\left(\frac{-y_{2}}{x_{1}}\right)-\operatorname{Li}_{2}\left(\frac{-x_{1}}{y_{2}}\right)-\operatorname{Li}_{2}\left(\frac{-y_{1}}{x_{2}}\right)\right. \\
+ & \left.\operatorname{Li}_{2}\left(\frac{b-a}{x_{2}}\right)+\operatorname{Li}_{2}\left(\frac{a-b}{y_{2}}\right)-\operatorname{Li}_{2}\left(\frac{b-a}{x_{1}}\right)-\operatorname{Li}_{2}\left(\frac{a-b}{y_{1}}\right)\right\},
\end{aligned}
$$

with

$$
\begin{aligned}
& x_{1,2}=\frac{1}{2}(1+b-a \pm \kappa), \\
& y_{1,2}=\frac{1}{2}(1+a-b \pm \kappa),
\end{aligned}
$$

and

$$
\kappa=\sqrt{1-2(a+b)+(a-b)^{2}} .
$$

Eq. (C.26) is more complicated than eq. (C.12), but leads to exactly the same numerical result. We have found it therefore a useful cross-check for our calculation.

Open Access. This article is distributed under the terms of the Creative Commons Attribution License (CC-BY 4.0), which permits any use, distribution and reproduction in any medium, provided the original author(s) and source are credited.

\section{References}

[1] Super-Kamiokande collaboration, Y. Fukuda et al., Evidence for oscillation of atmospheric neutrinos, Phys. Rev. Lett. 81 (1998) 1562 [hep-ex/9807003] [INSPIRE].

[2] SNO collaboration, Q.R. Ahmad et al., Direct evidence for neutrino flavor transformation from neutral current interactions in the Sudbury Neutrino Observatory, Phys. Rev. Lett. 89 (2002) 011301 [nucl-ex/0204008] [InSPIRE].

[3] KAmLAND collaboration, K. Eguchi et al., First results from KamLAND: evidence for reactor anti-neutrino disappearance, Phys. Rev. Lett. 90 (2003) 021802 [hep-ex/0212021] [INSPIRE].

[4] D.V. Forero, M. Tortola and J.W.F. Valle, Neutrino oscillations refitted, Phys. Rev. D 90 (2014) 093006 [arXiv: 1405.7540] [inSPIRE].

[5] F. Capozzi et al., Status of three-neutrino oscillation parameters, circa 2013, Phys. Rev. D 89 (2014) 093018 [arXiv: 1312.2878] [INSPIRE].

[6] M.C. Gonzalez-Garcia, M. Maltoni, J. Salvado and T. Schwetz, Global fit to three neutrino mixing: critical look at present precision, JHEP 12 (2012) 123 [arXiv: 1209.3023] [INSPIRE].

[7] S. Weinberg, Varieties of baryon and lepton nonconservation, Phys. Rev. D 22 (1980) 1694 [INSPIRE].

[8] E. Ma, Pathways to naturally small neutrino masses, Phys. Rev. Lett. 81 (1998) 1171 [hep-ph/9805219] [INSPIRE]. 
[9] P. Minkowski, $\mu \rightarrow e \gamma$ at a rate of one out of 1-billion muon decays?, Phys. Lett. B 67 (1977) 421 [INSPIRE].

[10] T. Yanagida, Horizontal symmetry and masses of neutrinos, Conf. Proc. C 7902131 (1979) 95 [INSPIRE].

[11] M. Gell-Mann, P. Ramond and R. Slansky, Complex spinors and unified theories, Conf. Proc. C 790927 (1979) 315 [arXiv:1306.4669] [INSPIRE].

[12] R.N. Mohapatra and G. Senjanović, Neutrino mass and spontaneous parity violation, Phys. Rev. Lett. 44 (1980) 912 [INSPIRE].

[13] J. Schechter and J.W.F. Valle, Neutrino masses in $\mathrm{SU}(2) \times \mathrm{U}(1)$ theories, Phys. Rev. D 22 (1980) 2227 [INSPIRE].

[14] M. Magg and C. Wetterich, Neutrino mass problem and gauge hierarchy, Phys. Lett. B 94 (1980) 61 [INSPIRE].

[15] R.N. Mohapatra and G. Senjanović, Neutrino masses and mixings in gauge models with spontaneous parity violation, Phys. Rev. D 23 (1981) 165 [INSPIRE].

[16] T.P. Cheng and L.-F. Li, Neutrino masses, mixings and oscillations in $\mathrm{SU}(2) \times \mathrm{U}(1)$ models of electroweak interactions, Phys. Rev. D 22 (1980) 2860 [INSPIRE].

[17] R. Foot, H. Lew, X.G. He and G.C. Joshi, Seesaw neutrino masses induced by a triplet of leptons, Z. Phys. C 44 (1989) 441 [inSPIRE].

[18] R.N. Mohapatra and J.W.F. Valle, Neutrino mass and baryon number nonconservation in superstring models, Phys. Rev. D 34 (1986) 1642 [INSPIRE].

[19] E.K. Akhmedov, M. Lindner, E. Schnapka and J.W.F. Valle, Left-right symmetry breaking in NJLS approach, Phys. Lett. B 368 (1996) 270 [hep-ph/9507275] [INSPIRE].

[20] E.K. Akhmedov, M. Lindner, E. Schnapka and J.W.F. Valle, Dynamical left-right symmetry breaking, Phys. Rev. D 53 (1996) 2752 [hep-ph/9509255] [INSPIRE].

[21] A. Zee, A theory of lepton number violation, neutrino Majorana mass and oscillation, Phys. Lett. B 93 (1980) 389 [Erratum ibid. B 95 (1980) 461] [INSPIRE].

[22] L. Wolfenstein, A theoretical pattern for neutrino oscillations, Nucl. Phys. B 175 (1980) 93 [INSPIRE].

[23] K.R.S. Balaji, W. Grimus and T. Schwetz, The solar LMA neutrino oscillation solution in the Zee model, Phys. Lett. B 508 (2001) 301 [hep-ph/0104035] [INSPIRE].

[24] X.-G. He, Is the Zee model neutrino mass matrix ruled out?, Eur. Phys. J. C 34 (2004) 371 [hep-ph/0307172] [INSPIRE].

[25] D. Aristizabal Sierra and D. Restrepo, Leptonic charged Higgs decays in the Zee model, JHEP 08 (2006) 036 [hep-ph/0604012] [INSPIRE].

[26] A. Zee, Quantum numbers of Majorana neutrino masses, Nucl. Phys. B 264 (1986) 99 [INSPIRE].

[27] K.S. Babu, Model of 'calculable' Majorana neutrino masses, Phys. Lett. B 203 (1988) 132 [INSPIRE].

[28] D. Choudhury, R. Gandhi, J.A. Gracey and B. Mukhopadhyaya, Two loop neutrino masses and the solar neutrino problem, Phys. Rev. D 50 (1994) 3468 [hep-ph/9401329] [INSPIRE]. 
[29] T. Kitabayashi and M. Yasue, Neutrino oscillations induced by two loop radiative mechanism, Phys. Lett. B 490 (2000) 236 [hep-ph/0006014] [INSPIRE].

[30] K.S. Babu and C. Macesanu, Neutrino masses and mixings in a minimal $\mathrm{SO}(10)$ model, Phys. Rev. D 72 (2005) 115003 [hep-ph/0505200] [INSPIRE].

[31] D. Aristizabal Sierra and M. Hirsch, Experimental tests for the Babu-Zee two-loop model of Majorana neutrino masses, JHEP 12 (2006) 052 [hep-ph/0609307] [INSPIRE].

[32] M. Nebot, J.F. Oliver, D. Palao and A. Santamaria, Prospects for the Zee-Babu model at the CERN LHC and low energy experiments, Phys. Rev. D 77 (2008) 093013 [arXiv:0711. 0483] [INSPIRE].

[33] D. Schmidt, T. Schwetz and H. Zhang, Status of the Zee-Babu model for neutrino mass and possible tests at a like-sign linear collider, Nucl. Phys. B 885 (2014) 524 [arXiv:1402.2251] [INSPIRE].

[34] J. Herrero-Garcia, M. Nebot, N. Rius and A. Santamaria, The Zee-Babu model revisited in the light of new data, Nucl. Phys. B 885 (2014) 542 [arXiv: 1402.4491] [InSPIRE].

[35] K.S. Babu and S. Nandi, Natural fermion mass hierarchy and new signals for the Higgs boson, Phys. Rev. D 62 (2000) 033002 [hep-ph/9907213] [INSPIRE].

[36] F. Bonnet, D. Hernandez, T. Ota and W. Winter, Neutrino masses from higher than $D=5$ effective operators, JHEP 10 (2009) 076 [arXiv:0907.3143] [INSPIRE].

[37] K.S. Babu, S. Nandi and Z. Tavartkiladze, New mechanism for neutrino mass generation and triply charged Higgs bosons at the LHC, Phys. Rev. D 80 (2009) 071702 [arXiv:0905.2710] [INSPIRE].

[38] F. Bonnet, M. Hirsch, T. Ota and W. Winter, Systematic study of the D $=5$ Weinberg operator at one-loop order, JHEP 07 (2012) 153 [arXiv:1204.5862] [INSPIRE].

[39] E. Ma, Verifiable radiative seesaw mechanism of neutrino mass and dark matter, Phys. Rev. D 73 (2006) 077301 [hep-ph/0601225] [INSPIRE].

[40] T. Hahn, Generating Feynman diagrams and amplitudes with FeynArts 3, Comput. Phys. Commun. 140 (2001) 418 [hep-ph/0012260] [INSPIRE].

[41] S.T. Petcov and S.T. Toshev, Conservation of lepton charges, massive Majorana and massless neutrinos, Phys. Lett. B 143 (1984) 175 [INSPIRE].

[42] K.S. Babu and E. Ma, Natural hierarchy of radiatively induced Majorana neutrino masses, Phys. Rev. Lett. 61 (1988) 674 [INSPIRE].

[43] E. Ma and J. Wudka, Vector-boson-induced neutrino mass, Phys. Lett. B 712 (2012) 391 [arXiv: 1202.3098] [INSPIRE].

[44] K. Bamba, C.Q. Geng and S.H. Ho, Radiative neutrino mass generation and dark energy, JCAP 09 (2008) 001 [arXiv:0806.0952] [INSPIRE].

[45] M. Lindner, D. Schmidt and T. Schwetz, Dark matter and neutrino masses from global $\mathrm{U}(1)_{B-L}$ symmetry breaking, Phys. Lett. B 705 (2011) 324 [arXiv:1105.4626] [INSPIRE].

[46] M. Aoki, S. Kanemura, T. Shindou and K. Yagyu, An R-parity conserving radiative neutrino mass model without right-handed neutrinos, JHEP 07 (2010) 084 [Erratum ibid. 11 (2010) 049] [arXiv: 1005.5159] [INSPIRE].

[47] M. Kohda, H. Sugiyama and K. Tsumura, Lepton number violation at the LHC with leptoquark and diquark, Phys. Lett. B 718 (2013) 1436 [arXiv:1210.5622] [INSPIRE]. 
[48] E. Ma, $Z_{3}$ dark matter and two-loop neutrino mass, Phys. Lett. B 662 (2008) 49 [arXiv:0708.3371] [INSPIRE].

[49] F. del Aguila, A. Aparici, S. Bhattacharya, A. Santamaria and J. Wudka, A realistic model of neutrino masses with a large neutrinoless double beta decay rate, JHEP 05 (2012) 133 [arXiv:1111.6960] [INSPIRE].

[50] G. Guo, X.-G. He and G.-N. Li, Radiative two loop inverse seesaw and dark matter, JHEP 10 (2012) 044 [arXiv: 1207.6308] [INSPIRE].

[51] G.-N. Li, G. Guo, B. Ren, Y.-J. Zheng and X.-G. He, Lepton number violation and $h \rightarrow \gamma \gamma$ in a radiative inverse seesaw dark matter model, JHEP 04 (2013) 026 [arXiv:1212.5528] [INSPIRE].

[52] D. Chang and H.N. Long, Interesting radiative patterns of neutrino mass in an $\mathrm{SU}(3)_{C} \times \mathrm{SU}(3)_{L} \times \mathrm{U}(1)_{X}$ model with right-handed neutrinos, Phys. Rev. D 73 (2006) 053006 [hep-ph/0603098] [INSPIRE].

[53] M. Aoki and T. Toma, Impact of semi-annihilation of $Z_{3}$ symmetric dark matter with radiative neutrino masses, JCAP 09 (2014) 016 [arXiv:1405.5870] [INSPIRE].

[54] H. Okada, T. Toma and K. Yagyu, Inert extension of the Zee-Babu model, Phys. Rev. D 90 (2014) 095005 [arXiv: 1408.0961] [INSPIRE].

[55] C.-S. Chen, C.Q. Geng and J.N. Ng, Unconventional neutrino mass generation, neutrinoless double beta decays and collider phenomenology, Phys. Rev. D 75 (2007) 053004 [hep-ph/0610118] [INSPIRE].

[56] C.-S. Chen, C.-Q. Geng, J.N. Ng and J.M.S. Wu, Testing radiative neutrino mass generation at the LHC, JHEP 08 (2007) 022 [arXiv:0706.1964] [INSPIRE].

[57] S.F. King, A. Merle and L. Panizzi, Effective theory of a doubly charged singlet scalar: complementarity of neutrino physics and the LHC, JHEP 11 (2014) 124 [arXiv:1406.4137] [INSPIRE].

[58] M. Gustafsson, J.M. No and M.A. Rivera, Radiative neutrino mass generation linked to neutrino mixing and $0 \nu \beta \beta$-decay predictions, Phys. Rev. D 90 (2014) 013012 [arXiv:1402.0515] [INSPIRE].

[59] M. Gustafsson, J.M. No and M.A. Rivera, Predictive model for radiatively induced neutrino masses and mixings with dark matter, Phys. Rev. Lett. 110 (2013) 211802 [arXiv:1212.4806] [INSPIRE].

[60] P.W. Angel, Y. Cai, N.L. Rodd, M.A. Schmidt and R.R. Volkas, Testable two-loop radiative neutrino mass model based on an $L L Q d^{c} Q d^{c}$ effective operator, JHEP 10 (2013) 118 [Erratum ibid. 11 (2014) 092] [arXiv: 1308. 0463] [INSPIRE].

[61] F. Borzumati and J.S. Lee, Novel constraints on $\Delta L=1$ interactions from neutrino masses, Phys. Rev. D 66 (2002) 115012 [hep-ph/0207184] [INSPIRE].

[62] P. Dey, A. Kundu, B. Mukhopadhyaya and S. Nandi, Two-loop neutrino masses with large $R$-parity violating interactions in supersymmetry, JHEP 12 (2008) 100 [arXiv:0808.1523] [INSPIRE].

[63] K.S. Babu and J. Julio, Two-loop neutrino mass generation through leptoquarks, Nucl. Phys. B 841 (2010) 130 [arXiv: 1006.1092] [INSPIRE]. 
[64] K.S. Babu and J. Julio, Radiative neutrino mass generation through vector-like quarks, Phys. Rev. D 85 (2012) 073005 [arXiv: 1112.5452] [INSPIRE].

[65] Y. Kajiyama, H. Okada and T. Toma, Multicomponent dark matter particles in a two-loop neutrino model, Phys. Rev. D 88 (2013) 015029 [arXiv:1303.7356] [INSPIRE].

[66] S. Baek, H. Okada and T. Toma, Two loop neutrino model and dark matter particles with global B-L symmetry, JCAP 06 (2014) 027 [arXiv: 1312.3761] [INSPIRE].

[67] E. Ma and U. Sarkar, Revelations of the $E_{6} / \mathrm{UN}(1)$ model: two-loop neutrino mass and dark matter, Phys. Lett. B 653 (2007) 288 [arXiv:0705.0074] [inSPIRE].

[68] M. Aoki, J. Kubo and H. Takano, Two-loop radiative seesaw mechanism with multicomponent dark matter explaining the possible $\gamma$ excess in the Higgs boson decay and at the Fermi LAT, Phys. Rev. D 87 (2013) 116001 [arXiv:1302.3936] [InSPIRE].

[69] W. Grimus and L. Lavoura, A neutrino mass matrix with seesaw mechanism and two loop mass splitting, Phys. Rev. D 62 (2000) 093012 [hep-ph/0007011] [INSPIRE].

[70] S. Davidson, G. Isidori and A. Strumia, The smallest neutrino mass, Phys. Lett. B 646 (2007) 100 [hep-ph/0611389] [INSPIRE].

[71] A.S. Joshipura and S.D. Rindani, Neutrino anomalies in an extended Zee model, Phys. Lett. B 464 (1999) 239 [hep-ph/9907390] [INSPIRE].

[72] D. Chang and A. Zee, Radiatively induced neutrino Majorana masses and oscillation, Phys. Rev. D 61 (2000) 071303 [hep-ph/9912380] [INSPIRE].

[73] T. Kitabayashi, Two loop radiative corrections to neutrino masses in $\mathrm{SU}(3)_{L} \times \mathrm{U}(1)_{N}$ gauge models, hep-ph/0010341 [INSPIRE].

[74] T. Kitabayashi, Comment on neutrino masses and oscillations in an $\mathrm{SU}(3)_{L} \times \mathrm{U}(1)_{N}$ model with radiative mechanism, Phys. Rev. D 64 (2001) 057301 [hep-ph/0103195] [INSPIRE].

[75] T. Kitabayashi and M. Yasue, Large mixing angle $M S W$ solution in an $\mathrm{SU}(3)_{L} \times \mathrm{U}(1)_{N}$ gauge model with two loop radiative mechanism, Phys. Lett. B 508 (2001) 85 [hep-ph/0102228] [INSPIRE].

[76] T. Kitabayashi and M. Yasue, Radiatively induced neutrino masses and oscillations in an $\mathrm{SU}(3)_{L} \times \mathrm{U}(1)_{N}$ gauge model, Phys. Rev. D 63 (2001) 095002 [hep-ph/0010087] [INSPIRE].

[77] Y. Kajiyama, H. Okada and K. Yagyu, Two loop radiative seesaw model with inert triplet scalar field, Nucl. Phys. B 874 (2013) 198 [arXiv: 1303.3463] [InSPIRE].

[78] H. Okada, Two loop induced Dirac neutrino model and dark matters with global U(1)' symmetry, arXiv: 1404.0280 [INSPIRE].

[79] Y. Farzan, S. Pascoli and M.A. Schmidt, Recipes and ingredients for neutrino mass at loop level, JHEP 03 (2013) 107 [arXiv:1208.2732] [INSPIRE].

[80] K.S. Babu and C.N. Leung, Classification of effective neutrino mass operators, Nucl. Phys. B 619 (2001) 667 [hep-ph/0106054] [INSPIRE].

[81] K.-W. Choi, K.S. Jeong and W.Y. Song, Operator analysis of neutrinoless double beta decay, Phys. Rev. D 66 (2002) 093007 [hep-ph/0207180] [INSPIRE].

[82] A. de Gouvêa and J. Jenkins, A survey of lepton number violation via effective operators, Phys. Rev. D 77 (2008) 013008 [arXiv:0708.1344] [INSPIRE]. 
[83] P.W. Angel, N.L. Rodd and R.R. Volkas, Origin of neutrino masses at the LHC: $\Delta L=2$ effective operators and their ultraviolet completions, Phys. Rev. D 87 (2013) 073007 [arXiv:1212.6111] [INSPIRE].

[84] F. del Aguila, A. Aparici, S. Bhattacharya, A. Santamaria and J. Wudka, Effective Lagrangian approach to neutrinoless double beta decay and neutrino masses, JHEP 06 (2012) 146 [arXiv: 1204.5986] [INSPIRE].

[85] J.A. Casas and A. Ibarra, Oscillating neutrinos and $\mu \rightarrow e, \gamma$, Nucl. Phys. B 618 (2001) 171 [hep-ph/0103065] [INSPIRE].

[86] D. Aristizabal Sierra, M. Hirsch and S.G. Kovalenko, Leptoquarks: neutrino masses and accelerator phenomenology, Phys. Rev. D 77 (2008) 055011 [arXiv:0710.5699] [InSPIRE].

[87] D. Aristizabal Sierra, J. Kubo, D. Restrepo, D. Suematsu and O. Zapata, Radiative seesaw: warm dark matter, collider and lepton flavour violating signals, Phys. Rev. D 79 (2009) 013011 [arXiv: 0808.3340] [INSPIRE].

[88] P. Fileviez Perez and M.B. Wise, On the origin of neutrino masses, Phys. Rev. D 80 (2009) 053006 [arXiv: 0906 .2950] [INSPIRE].

[89] Y. Cai, J.D. Clarke, M.A. Schmidt and R.R. Volkas, Testing radiative neutrino mass models at the LHC, JHEP 02 (2015) 161 [arXiv:1410.0689] [INSPIRE].

[90] D. Restrepo, O. Zapata and C.E. Yaguna, Models with radiative neutrino masses and viable dark matter candidates, JHEP 11 (2013) 011 [arXiv:1308.3655] [INSPIRE].

[91] K.L. McDonald and B.H.J. McKellar, Evaluating the two loop diagram responsible for neutrino mass in Babu's model, hep-ph/0309270 [INSPIRE].

[92] J. van der Bij and M.J.G. Veltman, Two loop large Higgs mass correction to the $\rho$ parameter, Nucl. Phys. B 231 (1984) 205 [inSPIRE].

[93] G. Passarino and M.J.G. Veltman, One loop corrections for $e^{+} e^{-}$annihilation into $\mu^{+} \mu^{-}$in the Weinberg model, Nucl. Phys. B 160 (1979) 151 [INSPIRE]. 